\title{
Regulating peroxisome-ER contacts via the ACBD5-VAPB tether by FFAT motif phosphorylation and GSK3 $\beta$
}

Suzan Kors ${ }^{1}$, Christian Hacker ${ }^{1}$, Chloe Bolton ${ }^{1}$, Renate Maier ${ }^{2}$, Lena Reimann ${ }^{2}$, Emily J.A. Kitchener ${ }^{1}$, Bettina Warscheid ${ }^{2,3}$, Joseph L. Costello ${ }^{1 \#}$, Michael Schrader ${ }^{1 \#}$

${ }^{1}$ College of Life and Environmental Sciences, Biosciences, University of Exeter, EX4 4QD Exeter, UK ${ }^{2}$ Institute of Biology II, Biochemistry and Functional Proteomics, Faculty of Biology, University of Freiburg, 79104 Freiburg, Germany

${ }^{3}$ CIBSS Centre for Integrative Biological Signalling Studies, University of Freiburg, 79104 Freiburg, Germany

Correspondence to Michael Schrader: M.Schrader@exeter.ac.uk, Joseph Costello:

\section{J.Costello@exeter.ac.uk}

\# J. Costello and M. Schrader contributed equally to this paper.

Running title: Phosphorylation regulates the ACBD5-VAPB tether

Keywords: peroxisomes; membrane contact sites; FFAT motif; phosphorylation; GSK3 $\beta$ 


\section{SUMMARY}

Kors et al. reveal that peroxisome-ER associations via the ACBD5-VAPB tether are regulated by phosphorylation and GSK3 $\beta$ in mammalian cells. Phosphorylation sites in the FFAT-like motif of ACBD5 affect the binding to VAPB and thus, peroxisome-ER contact sites, differently.

\section{ABSTRACT}

Peroxisomes and the endoplasmic reticulum (ER) cooperate in cellular lipid metabolism. They form membrane contacts through interaction of the peroxisomal membrane protein ACBD5 [acylcoenzyme A-binding domain protein 5] and the ER-resident protein VAPB [vesicle-associated membrane protein-associated protein $B]$. ACBD5 binds to the major sperm protein domain of VAPB via its FFAT-like [two phenylalanines (FF) in an acidic tract] motif. However, molecular mechanisms, which regulate formation of these membrane contact sites, are unknown. Here, we reveal that peroxisome-ER associations via the ACBD5-VAPB tether are regulated by phosphorylation. We show that ACBD5-VAPB binding is phosphatase-sensitive and identify phosphorylation sites in the flanking regions and core of the FFAT-like motif, which alter interaction with VAPB and thus, peroxisome-ER contact sites differently. Moreover, we demonstrate that GSK3 $\beta$ [glycogen synthase kinase-3 beta] regulates this interaction. Our findings reveal for the first time a molecular mechanism for the regulation of peroxisome-ER contacts in mammalian cells and expand the current model of FFAT motifs and VAP interaction. 


\section{INTRODUCTION}

Peroxisomes are small, single membrane bound organelles with key roles in cellular lipid and hydrogen peroxide metabolism. They contribute to a wide range of metabolic processes including the $\beta$ oxidation of fatty acids and the synthesis of bile acids and plasmalogens (myelin sheath lipids) (Wanders and Waterham, 2006). To fulfil those functions, peroxisomes interact and cooperate with other organelles, such as the endoplasmic reticulum (ER), mitochondria, lysosomes and lipid droplets, in order to efficiently transfer lipid metabolites (e.g. plasmalogen intermediates, chain-shortened acylCoAs, cholesterol and very long chain fatty acids (VLCFAs), respectively) (Chu et al., 2015; Wanders et al., 2016; Shai et al., 2018; Chang et al., 2019).

This collaboration requires close proximity of the organelles, which is mediated by protein tethering complexes that physically bridge apposing organelles (Prinz et al., 2020; Silva et al., 2020). Recently, we and others identified novel tethering complexes that mediate membrane contacts between peroxisomes and the ER in mammalian cells (Costello et al., 2017b; c; Hua et al., 2017; Xiao et al., 2019; Guillén-Samander et al., 2021). We revealed that the peroxisomal acyl-CoA binding domain proteins ACBD4 and ACBD5 interact with ER-resident VAMP-associated proteins (VAPs), a protein family widely involved in tethering the ER to other organelles (Murphy and Levine, 2016). This interaction involves a FFAT [two phenylalanines (FF) in an acidic tract] -like motif within ACBD4/5 and the VAP MSP [major sperm protein] domain (Costello et al., 2017b; Hua et al., 2017). Peroxisome-ER contacts control peroxisome movement and positioning, the delivery of lipids required for peroxisomal membrane expansion prior to division and the transfer of lipid metabolites such as cholesterol, plasmalogens and VLCFAs, although the latter has yet to be formally demonstrated (Darwisch et al., 2020; Schrader et al., 2020).

In general, although the overall pattern of membrane contact sites between organelles has been shown to be relatively stable, individual organelle contacts are dynamic (Valm et al., 2017), suggesting that protein tethers between organelles are highly regulated. The importance of dynamism in organelle contacts is exemplified by the mitochondria-ER-cortex tether in yeast which needs to be remodelled during meiotic divisions (Sawyer et al., 2019). This is achieved by rapid degradation of the organelle tethering complex allowing mitochondrial detachment. Peroxisome-ER contacts also need to be dynamic in order to modulate peroxisome movement and positioning, as well as metabolite flow between the organelles. However, knowledge on how the majority of membrane contact sites are regulated is limited. Previous studies found that several proteins present at membrane contact sites can be regulated by phosphorylation, including lipid transfer proteins CERT, OSBP and ORP3 (Goto et al., 2012; Kumagai et al., 2014; Weber-Boyvat et al., 2015). These proteins have in common that they bind to the MSP domain of VAP via their FFAT motif. This motif consists of the core consensus sequence ${ }^{1}$ EFFDA-E ${ }^{7}$ flanked by a stretch of acidic residues (Loewen et al., 2003). Numerous proteins which possess potential FFAT motifs have now been identified (Slee and Levine, 2019), and previous work has implicated phosphorylation of particular residues in the FFAT motif in regulating VAP binding (Kumagai et al., 2014; Kirmiz et al., 2018; Johnson et al., 2018). Recent studies revealed that VAP can also bind to unconventional FFAT motifs with a serine/threonine residue at position 4, but only when this residue is phosphorylated (phospho-FFAT) to mimic the acidic amino acid present in the conventional motif (Kirmiz et al., 2018; Di Mattia et al., 2020; Guillén-Samander et al., 2021). However, a thorough understanding of the regulation of FFAT-VAP interactions by phosphorylation and how this 
is linked to kinases and phosphatases as well as other signalling networks is still lacking (Mikitova and Levine, 2012; Murphy and Levine, 2016).

Here, we reveal that peroxisome-ER associations via the ACBD5-VAPB tether are regulated by phosphorylation. We show that the ACBD5-VAPB interaction is phosphatase-sensitive and that ACBD5 phosphomimetic and non-phosphorylatable mutants influence interaction with VAPB and thus, peroxisome-ER contact sites differently. Moreover, we show that glycogen synthase kinase-3 beta (GSK3 $\beta$ ) associates with the ACBD5-VAPB complex to regulate peroxisome-ER contacts. GSK3 $\beta$ can directly phosphorylate ACBD5 in the FFAT core at serine-269 (S269), a residue that, when phosphorylated, blocks binding to VAPB. Our findings reveal for the first time a molecular mechanism for the regulation of peroxisome-ER contacts in mammalian cells, provide one of the first clear examples for a physiological role of phosphorylation of peroxisomal proteins in mammals, and expand the current model of FFAT motifs and VAP interaction.

\section{RESULTS}

\section{The overall phosphorylation status of ACBD5, but not ACBD4, affects VAPB binding}

Several studies have suggested a general role for phosphorylation in regulating FFAT-VAP interactions, and multiple phosphorylation sites in ACBD4 and ACBD5 have been reported in high-throughput studies (Bian et al., 2014; Sharma et al., 2014). To confirm ACBD4/5 phosphorylation and to study its role in the interaction between ACBD4 and ACBD5 with VAPB, we first investigated whether ACBD4 and ACBD5 were phosphorylated using the Phos-tag system (Kinoshita et al., 2006). Altered migration in Phos-tag SDS-PAGE following phosphatase treatment indicated that both ACBD4 and ACBD5 were phosphorylated (Fig. 1A) as suggested (Bian et al., 2014; Sharma et al., 2014). Next, we tested whether phosphorylation of ACBD4 and ACBD5 had an effect on VAPB binding. COS-7 cells expressing FLAGACBD4 or FLAG-ACBD5 were lysed in the presence of phosphatase inhibitor, or lysates were treated with $\lambda$ protein phosphatase ( $\lambda P P$ ) (as in Fig. 1A). The samples were then incubated with recombinant GST-VAPB, expressed and purified from E. coli. Interaction of GST-VAPB with FLAG-ACBD4 was similar in phosphatase inhibitor and phosphatase-treated, immunoprecipitated samples, indicating that the ACBD4-VAPB binding is insensitive to phosphatase treatment (Fig. 1B). In contrast, $\lambda$ PP-treated FLAGACBD5 failed to bind GST-VAPB (Fig. 1B). A FFAT-mutant (mFFAT) of FLAG-ACBD5, which was previously shown to abolish VAPB interaction, was used as a negative control (Costello et al., 2017b). A FFAT mutation in ACBD4 (ACBD4 mFFAT) also resulted in loss of association with GST-VAPB, confirming that, similar to ACBD5, a single FFAT-like motif in ACBD4 is essential for VAPB binding (Fig. 1B). Similar results were obtained with endogenous VAPB in a binding assay using lysates of COS-7 cells expressing tagged versions of ACBD4 or ACBD5 (Fig. 1C, Fig. S1A). Binding of Myc-ACBD5 to endogenous VAPB was significantly reduced upon $\lambda$ PP-treatment (Fig. 1C). We conclude that the interaction between $A C B D 5$ and $V A P B$, but not ACBD4-VAPB, is highly sensitive to phosphatase treatment.

\section{ACBD5 phosphorylation profile}

To determine the potential phosphorylation sites that are responsible for the phosphatase sensitivity of the ACBD5-VAPB interaction we combined a database search (Hornbeck et al., 2015; Ullah et al., 2016) with our own phosphorylation analysis of ACBD5 by mass spectrometry (MS) (Fig. 1D). Multiple phosphorylation sites were identified, but an initial mutational analysis of prominent phosphorylation sites in ACBD5 did not identify any residues involved in VAPB binding (Fig. S1B). A more detailed 
analysis of our MS phosphorylation data revealed that interestingly, there was an apparent gap in the peptide coverage of ACBD5, which included the FFAT-like motif and surrounding region (Fig. 1D). As this stretch contained multiple serine/threonine residues and the VAPB binding site, we decided to explore this region by placing it in ACBD4 (Fig. S2A). Replacing the FFAT-like motif of ACBD4 with that of $A C B D 5$ now rendered the interaction between ACBD4 and VAPB sensitive to $\lambda$ PP-treatment (Fig. 1E). This suggests that phosphorylation in and around the FFAT-like motif of ACBD5 is responsible for the sensitivity of the ACBD5-VAPB interaction to phosphatase treatment. To investigate how phosphorylation of this region can affect VAPB binding, we next generated phosphosite mutants of the highly conserved residues (Fig. 2A, Fig. S2B-D).

\section{Non-phosphorylatable residues in the acidic tract reduce ACBD5-VAPB binding}

The core FFAT motif ( ${ }^{1}$ EFFDA- $\left.E^{7}\right)$ is often surrounded by acidic residues (acidic tract herein), which are thought to contribute to the initial interaction with VAPB as part of a two-step binding model (Fig. 2B). The presence of serine/threonine residues in the acidic tract of FFAT(-like) motifs is a common feature (Loewen et al., 2003; Murphy and Levine, 2016), including that of ACBD5 (Fig. 2A). Phosphorylation of these residues could mimic canonical aspartic acid and glutamic acid residues, aiding the acidic environment and potentially increasing binding to VAPB. To test this, we generated Myc-tagged single, double and triple ACBD5 phosphosite mutants of the acidic tract by mutating threonine-258 (T258), serine-259 (S259), S261 and S263 to alanine (A), to mimic non-phosphorylated forms. All phosphomutants were properly targeted to peroxisomes (Fig. S3A), as the peroxisome targeting signal located in the transmembrane domain and tail of ACBD4/5 was not altered (Costello et al., 2017a).

The ACBD5 phosphosite mutants were expressed in COS-7 cells and interaction with endogenous VAPB was assessed by immunoprecipitation (Fig. 2C). Whereas the S259A single mutant did not significantly reduce VAPB binding, the S261A and S263A single mutants showed a significant reduction, suggesting that phosphorylation of these serine residues in the acidic tract is important for VAPB interaction (Fig. 2C). The double mutants T258A/S259A and S259A/S261A also caused a reduction in VAPB binding when compared to WT control, whereas the S261A/S263A double and S259A/S261A/S263A triple mutants had the most prominent effect. This suggests that (i) residues S261 and S263 are the most significant for VAPB binding, and (ii) the overall acidity of the acidic tract contributes to VAPB interaction.

The acidic tract of ACBD5 contributes to the phosphatase-sensitivity of the ACBD5-VAPB interaction Overall, our results indicate that lack of phosphorylation of residues in the acidic tract of ACBD5 reduces its binding to VAPB. Therefore, we hypothesized that phosphomimetic mutation of these residues would overcome the sensitivity of the ACBD5-VAPB interaction for phosphatase treatment (Fig. 1C). We generated Myc-ACBD5 single and triple site phosphomimetic mutants by replacing S259, S261 and S263 with glutamic acid (E), and assessed their binding to endogenous VAPB by immunoprecipitation after $\lambda$ PP-treatment of the cell lysates (Fig. 2D, E). We observed that binding of the single mutants S259E and S263E to VAPB was still reduced upon $\lambda$ PP-treatment, comparable to WT control (Fig. 2D). However, the single S261E and triple S259E/S261E/S263E phosphomimetic mutants showed significantly more binding to VAPB than wild type following $\lambda P P$-treatment (Fig. 2D, E). This confirms that phosphorylation of the acidic tract contributes to VAPB binding, but also indicates that additional, as yet uncharacterised phosphatase-sensitive elements exist which mediate VAPB binding. 


\section{Additional phospho-mutants of the FFAT-like motif region affect VAPB binding}

ACBD5 contains additional serine/threonine residues further upstream of the FFAT-like motif and within the FFAT core, which have been found to be phosphorylated in individual high throughput studies (Wang et al., 2008; Bian et al., 2014) (Fig. 1D). To determine whether the phosphorylation of ACBD5 at T252 (upstream of the FFAT-like motif) and S269 (within the FFAT core) affects ACBD5-VAPB interaction, we generated Myc-ACBD5 non-phosphorylatable and phosphomimetic site mutants by replacing these residues with alanine $(A)$ or glutamic acid $(E)$, respectively. The constructs were expressed in COS-7 cells and their interaction with endogenous VAPB was assessed by immunoprecipitation (Fig. 3A). Both the T252A and T252E mutant of Myc-ACBD5 bound VAPB similar to WT control levels indicating that upstream T252 phosphorylation/dephosphorylation is not crucial for ACBD5-VAPB binding. The phosphomimetic mutation of the serine in the FFAT core of ACBD5 (S269E; position 5) abolished binding to VAPB, whereas ACBD5 S269A still co-precipitated with VAPB (Fig. 3A).

Serine residues at similar positions in ACBD4 were investigated in parallel (Fig. S2B). Phosphomimetic site mutants of residues upstream of the FFAT-like motif of FLAG-ACBD4 (S166E/S169E/S171E) coprecipitated more prominently with VAPB than the FLAG-ACBD4 non-phosphorylatable mutants (S166A/S169A/S171A) when compared to WT controls (Fig. S3B, C). Overall, the non-phosphorylatable ACBD4 mutants appeared to have little impact on VAPB binding, whereas the ACBD4 phosphomimetic mutants increased interaction. The phosphomimetic mutant of the serine at position 5 of the ACBD4 FFAT core (S183) showed, similar to this residue in ACBD5, loss of VAPB binding (Fig. S3B).

\section{Serine $\mathbf{2 6 9}$ in the FFAT core of ACBD5 is phosphorylated}

Our results indicate that phosphorylation of the serine in the FFAT core would abolish VAPB binding (Fig. 3A, B). To confirm that this serine in the FFAT core of ACBD5 can be phosphorylated under our experimental conditions, we generated a phospho-specific antibody towards ACBD5 pS269 (Eurogentec). As the antibody was not able to recognize ACBD5 in whole cell lysates, ACBD5 was immunoprecipitated to test the specificity of the antibody. The ACBD5 pS269 antibody showed a reduced signal in phosphatase treated lysates, while the signal of the ACBD5 control antibody (generated against a peptide of the same region), was not affected by the treatment (Fig. 3C). To further validate the phospho-antibody, COS-7 cells were transfected with Myc-ACBD5 WT or S269A phosphosite mutant. The ACBD5 control antibody detected both the ACBD5 WT and mutant forms, however, the pS269 antibody was not able to detect the site-specific mutant (Fig 3D). These experiments indicate that the generated ACBD5 pS269 antibody is specific and that the serine in the FFAT core of ACBD5 can be phosphorylated in COS-7 cells.

We now used the ACBD5 pS269 antibody to assess whether phosphorylation of the serine at position 5 of the ACBD5 FFAT core (S269) would inhibit VAPB binding. We hypothesised that only a certain fraction of ACBD5 would be phosphorylated at S269, that this population would not interact with VAPB, and thus, in an ACBD5-VAPB interaction assay the phosphorylated form would be enriched in the non-bound fraction (Fig. 3E). To investigate this using ourpS269 antibody (which cannot detect ACBD5 in whole cell lysates), we expressed Myc-VAPB and FLAG-ACBD5 separately in COS-7 cells. Both proteins were immunoprecipitated, and subsequently incubated together to allow Myc-VAPB and FLAG-ACBD5 to interact. We then compared the phosphorylation state of FLAG-ACBD5 bound to Myc-VAPB with the non-bound fraction. Incubation with a FLAG antibody revealed approximately equal amounts of FLAG-ACBD5 in both the bound and non-bound fraction (Fig. 3E). However, the S269 phosphorylated form of ACBD5 was barely detectable in the 
VAPB-bound fraction, and was instead enriched in the non-bound fraction. This indicates that phosphorylation of ACBD5 at S269 in the FFAT core inhibits the interaction with VAPB.

\section{Phosphosites within the ACBD5 FFAT-like motif influence peroxisome-ER contacts}

We next investigated if phosphorylation of the ACBD5 FFAT-like motif, which modulates ACBD5-VAPB binding, could also alter peroxisome-ER interactions in mammalian cells. We expressed a set of MycACBD5 phosphosite mutants in ACBD5 knock-out (KO) HeLa cells and quantified peroxisome-ER association by transmission electron microscopy using unbiased spatial stereology as previously described (Costello et al., 2017b) (Fig. 4A-C). We have recently shown that ACBD5 KO HeLa cells have reduced peroxisome-ER associations (Bishop et al., 2019). Restoration of peroxisome-ER contacts upon ACBD5 expression was quantified by determining the mean population of peroxisomes in close contact ( $<15 \mathrm{~nm}$ ) with the ER (Fig. 4B, mean attachment) and the proportion of the peroxisome surface closely apposed ( $<15 \mathrm{~nm}$ ) to the ER (Fig. 4C, mean ER contact) (Costello et al., 2017b). We observed that expression of Myc-ACBD5 WT restored peroxisome-ER associations in ACBD5 KO cells to a level comparable to control HeLa cells (Bishop et al., 2019) (Fig. 4A-C). The S259A mutant, which did not affect ACBD5-VAPB interaction (Fig. 2C), restored peroxisome-ER contacts similar to ACBD5 WT, whereas expression of mutants which significantly reduced or abolished VAPB binding (nonphosphorylatable S261A and S259A/S261A/S263A, phosphomimetic S269E) (Fig. 2C, 3A) did not restore peroxisome-ER associations (Fig. 4A-C). All constructs were expressed equally well in ACBD5 KO cells (Fig. 4D). We have shown previously that the ACBD5-VAPB interaction plays a role in peroxisomal membrane expansion, which is a prerequisite for peroxisome division and multiplication (Schrader et al., 2016; Costello et al., 2017b). Peroxisome-ER tethering likely allows transfer of membrane lipids to promote peroxisome elongation (Schrader et al., 2020). In line with this, expression of ACBD5 WT in COS-7 cells induced the formation of tubular peroxisomes (Fig. S3A, D) (Hua et al., 2017). Interestingly, expression of ACBD5 S269E, which inhibits VAPB interaction and thus, peroxisome-ER contacts, did not promote peroxisome elongation. These observations further support the notion that ACBD5-VAPB mediated peroxisome-ER contacts support membrane lipid transfer by a yet unknown mechanism. Overall, this demonstrates that alterations in phosphorylated residues of ACBD5, which affect VAPB binding, also alter peroxisome-ER associations and peroxisome membrane dynamics.

\section{GSK3 $\beta$ alters the ACBD5-VAPB interaction}

To identify kinases/phosphatases involved in the phosphorylation/dephosphorylation of ACBD5 and thus, in regulating the ACBD5-VAPB interaction, we took a candidate-based approach with focus on known associations with $\mathrm{ACBD} 5$ or VAPB, and previous links to peroxisome function. This approach identified glycogen synthase kinase-3 beta (GSK3 $\beta$ ), which has recently been linked to regulation of peroxisome number in a Drosophila screening approach (Graves et al., 2020). GSK3 $\beta$ has also been linked to the regulation of PTPIP51-VAPB interaction, which forms a mitochondria-ER tethering complex (Stoica et al., 2014, 2016). To investigate a potential role for GSK3 $\beta$ in regulating the ACBD5VAPB interaction, we co-expressed GSK3 $\beta$ and Myc-VAPB in COS-7 cells and determined alterations in the interaction of Myc-VAPB with endogenous ACBD5 and PTPIP51 (Fig. 5A). We confirmed that GSK3 $\beta$ expression increased GSK3 $\beta$ 's activity and downstream signalling events (e.g. $\beta$-catenin phosphorylation and degradation) (Fig. S4A), and reduced the VAPB-PTPIP51 interaction as previously shown (Stoica et al., 2014) (Fig. 5A). The interaction between ACBD5 and VAPB was also significantly reduced, suggesting that GSK3 $\beta$ expression altered the interaction. 
To further elucidate the role of GSK3 $\beta$ in regulating the ACBD5-VAPB interaction, we treated HEK293T cells expressing FLAG-ACBD5 with the GSK3 $\beta$ inhibitor CHIR99021 (CHIR). Inhibition of GSK3 $\beta$ by CHIR was confirmed by decreased GSK3 $\beta$ Y 216 phosphorylation and stabilization of its substrate $\beta$-catenin (Fig. 5B). Addition of CHIR significantly increased the interaction between FLAG-ACBD5 and endogenous VAPB, further indicating a role for GSK3 3 in regulating ACBD5-VAPB binding.

\section{GSK3 $\beta$ associates with ACBD5 and VAPB}

To test for interaction of GSK3 $\beta$ with the ACBD5-VAPB complex, we immunoprecipitated VAPB and identified GSK3 $\beta$ as a potential binding partner (Fig. 5A), suggesting that GSK3 $\beta$ is present in a complex with VAPB and potentially ACBD5. We checked the protein sequence of GSK3 $\beta$ for potential FFAT

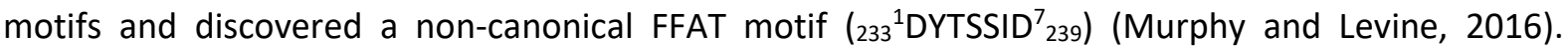
Although the predicted FFAT motif in GSK3 $\beta$ has a relatively low FFAT score (3.5; similar to ACBD4) and is located in a structured region, we generated a FFAT mutant (S237E 'mFFAT') which should abolish potential VAPB interaction. Expression of GSK3 $\beta$ S237E together with FLAG-VAPB showed that the co-immunoprecipitation of GSK3 $\beta$ with VAPB did not depend on the potential FFAT motif (Fig. 6A). To explore if GSK3 $\beta$ also associates with ACBD5, and if this interaction depends on VAPB, we coexpressed GSK3 $\beta$ and FLAG-ACBD5 WT, mFFAT or $\triangle T M D$, a mutant with cytosolic localisation (Fig. S3A) in COS-7 cells. GSK3 $\beta$ was immunoprecipitated with all FLAG-ACBD5 variants (Fig. 6A), indicating that the interaction of GSK3 $\beta$ with ACBD5 does not depend on ACBD5-VAPB binding, the presence of ACBD5 at ER contact sites, or ACBD5 anchorage at the peroxisomal membrane. Next, we assessed if the VAPB-GSK3 $\beta$ co-immunoprecipitation was dependent on VAPB's ability to bind to FFAT motifcontaining proteins such as ACBD5. We observed that a FLAG-VAPB mutant unable to bind FFAT-motifs (K87D/M89D mMSP (Kaiser et al., 2005); Fig. 6B, Fig. S4B) still immunopreciptated with GSK3 $\beta$ (Fig. 6B). Overall, these experiments show that both ACBD5 and VAPB immunoprecipitate GSK3 $\beta$, independent of their ability to interact with each other.

\section{Non-phosphorylatable residues in the acidic tract reduce ACBD5 S269 phosphorylation}

A dependence of the FFAT-VAP affinity on a combination of (non)phosphorylated residues/regions in and outside the FFAT region has been suggested, which involves 'crosstalk' of those sites in the regulation of protein interaction and function (Goto et al., 2012; Kumagai et al., 2014). Furthermore, (de)phosphorylated regions could also be binding sites for phosphatases and kinases that act on residues further up/downstream. Therefore, we decided to assess the phosphorylation status of the serine in the FFAT core (S269) of ACBD5 in the presence of non-phosphorylatable residues in the FFAT acidic tract (Fig. 6C). As the acidic tract of ACBD5 resembles the consensus sequence of GSK3 $\beta$ (SxxxpS) (Frame and Cohen, 2001), the binding to GSK3 was also examined. Both FLAG-ACBD5 S259A/S261A and S259A/S261A/S263A showed a strong reduction in S269 phosphorylation, whereas binding to coexpressed GSK3 $\beta$ was not significantly altered (Fig. 6 C). We suggest that residues in the acidic tract of ACBD5 are involved in regulating the phosphorylation of the FFAT core.

\section{GSK3 $\beta$ modulates the ACBD5-VAPB interaction via $\mathbf{5 2 6 9}$}

As both GSK3 $\beta$ activity and ACBD5 S269 phosphorylation inhibit the ACBD5-VAPB interaction, we explored the possibility that GSK3 $\beta$ modulates the interaction via S269. We co-expressed GSK3 $\beta$ or a catalytically inactive mutant (GSK3 3 K85A) (Fig. S4A) with Myc-ACBD5 WT or S269A in COS-7 cells, and assessed the binding of endogenous VAPB to Myc-ACBD5 by immunoprecipitation. The binding of VAPB to Myc-ACBD5 WT was significantly reduced in the presence of GSK3 $\beta$ WT compared to GSK3 $\beta$ 
K85A (Fig. 7A). However, this reduction in VAPB binding was restored with the ACBD5 mutant S269A, suggesting that the ability of GSK3 $\beta$ to modulate ACBD5-VAPB interaction was dependent on the presence of a serine at position 269. To assess whether the inhibition of VAPB binding by GSK3 $\beta$ is linked to the phosphorylation of this serine, we next analysed the levels of ACBD5 pS269. Expression of GSK3 $\beta$ WT significantly increased the pS269 levels, suggesting that GSK3 $\beta$ inhibits the ACBD5-VAPB interaction by inducing phosphorylation at S269 of ACBD5 (Fig. 7A). To examine whether GSK3 $\beta$ potentially phosphorylates S269 directly, we developed an in vitro kinase assay using recombinant protein. The level of $\mathbf{S} 269$ phosphorylation of recombinant ACBD5 was determined in the absence and presence of recombinant GSK3 3 . The pS269 antibody signal was highly increased in the presence of GSK3 $\beta$, showing that GSK3 $\beta$ can phosphorylate ACBD5 at this serine residue (Fig. 7B).

To explore if the inhibition of the ACBD5-VAPB interaction by GSK3 $\beta$ also alters peroxisome-ER membrane contacts, we expressed GSK3 $\beta$ and a catalytically inactive mutant (GSK3 $\beta$ K85A) in COS-7 cells and quantified peroxisome-ER association by transmission electron microscopy as described above (see Fig. 4). The proportion of peroxisome surface in contact with the ER was significantly reduced upon GSK3 $\beta$ WT expression (Fig. 7C, D, mean ER contact). Additionally, the number of peroxisomes in close contact with the ER also showed a slight, although not significant, reduction (Fig. 7D, mean attachment). We have previously shown that reduced ACBD5-VAPB interactions impact peroxisome membrane elongation in cells with impaired peroxisome division, likely because of reduced membrane lipid transport from the ER to peroxisomes (Costello et al., 2017b; Hua et al., 2017). To further investigate a role for GSK3 $\beta$ in inhibiting peroxisome-ER contacts, we expressed GSK3 $\beta$ in patient fibroblasts deficient in division factor MFF, which show highly elongated peroxisomes (Koch et al., 2016) (Fig. S4C). GSK3 $\beta$ significantly decreased the formation of these highly elongated peroxisomes, suggesting a change in peroxisome-ER membrane contacts (Fig. S4C). Together, these results implicate that GSK3 $\beta$ inhibits the ACBD5-VAPB interaction and reduces peroxisome-ER associations, required for peroxisomal membrane growth.

\section{DISCUSSION}

We show here that peroxisome-ER association via the ACBD5-VAPB tether is regulated by phosphorylation. Several lines of evidence support this: (a) ACBD5-VAPB interaction is phosphatasesensitive; (b) ACBD5 phosphomimetic and non-phosphorylatable mutants alter the interaction with VAPB; (c) ACBD5 phosphosite mutants impact peroxisome-ER interaction in mammalian cells; (d) GSK3 $\beta$ regulates the ACBD5-VAPB interaction, hence peroxisome-ER contacts; and (e) ACBD5 can be phosphorylated by GSK3 $\beta$. In conclusion, our findings reveal for the first time a molecular mechanism for the regulation of peroxisome-ER contacts in mammalian cells (Fig. 8).

Our findings also expand the current view and molecular understanding of FFAT motifs in general. We demonstrate that, as suggested in the original study describing FFAT motifs (Loewen et al., 2003), residues upstream of the canonical FFAT core are relevant for FFAT-VAP interactions. We show that phosphorylation of serine/threonine residues within the acidic tract (e.g. ACBD5 S261; Fig. 2C-E) or further extension of the acidic tract by upstream serine/threonine residues (e.g. ACBD4; Fig. S3B, C) improves binding to VAPB. The interaction of the FFAT motif with VAPB is thought to occur in two steps: an initial electrostatic interaction between the acidic tract upstream of the FFAT core and the basic electropositive face of the MSP domain of VAPB, followed by the binding of the FFAT core region to specific residues of the MSP domain (Furuita et al., 2010) (Fig. 2B; Fig. 8). Our findings suggest that the FFAT motif can be "activated" by adding a negatively charged phosphate group to 
serine/threonine residues, improving binding of the acidic tract to the basic electropositive face of the MSP domain. Our data on ACBD4 phosphorylation suggest that these residues can also potentially be located further upstream, thus extending the canonical FFAT-like motif, as the ACBD4 phosphomimetic mutants increased ACBD4-VAPB binding (Fig. S3B, C). These residues have been found to be phosphorylated in high-throughput studies (Hornbeck et al., 2015). However, in our experimental system non-phosphorylatable mutants (S166A/S169A/S171A) (Fig. S3B, C) and phosphatase treatment (Fig. 1B, Fig. S1A) did not have a significant impact on ACBD4-VAPB binding, suggesting low phosphorylation levels in our experimental setup. We therefore propose that the extended site may function as a switch to modulate ACBD4-VAPB binding potentially dependent on particular cellular conditions.

Numerous FFAT motif-containing proteins possess serine/threonine residues in their acidic tract, including ceramide transport protein CERT, lipid transfer protein STARD3 and potassium channel KV2. Phosphorylation of a single serine residue (S315) in the acidic tract of CERT has been reported to enhance VAPA interaction (Kumagai et al., 2014). However, mutation of this residue to S315A did not have much impact on VAPA interaction. The interaction between STARD3 FFAT peptide and endogenous VAPA/B was strengthened by phosphorylation of a serine residue in both the upstream and downstream flank of the acidic tract (Di Mattia et al., 2020). Kv2 contains a FFAT motif that without phosphorylation would not have an 'acidic tract' (Kirmiz et al., 2018; Johnson et al., 2018). Another example of such a protein is Chlamydia trachomatis IncV which contains 'acidic tracts' exclusively composed of serine residues (Stanhope et al., 2017). This suggests that overall phosphorylation is a general mechanism to "activate" the acidic tract and by doing so adjust VAPinteractions. As there are a large number of VAP interactors, and evidence of competition between FFAT proteins, these small increases in VAP affinity may have a significant impact. The importance of the residues in the acidic tract can also be illustrated by the identification of the unconventional FFATrelated FFNT [two phenylalanines (FF) in a neutral tract] motifs, that preferably bind to ER-resident MOSPD1/3 proteins that, like VAPA/B, possess a MSP domain (Cabukusta et al., 2020). Moreover, PTPIP51 contains an 'acidic tract' mainly composed of serine/threonine residues and was able to bind all VAP and MOSPD proteins. (De)phosphorylation of the residues in the tract could possibly switch the tract from acidic to neutral and vice versa, changing the affinity of PTPIP51 for VAPA/B and MOSPD1/3. However, our data also imply that there are potentially additional phosphorylation events involved in the regulation of the ACBD5-VAPB interaction. Phosphorylation of VAPB may also influence ACBD5-VAPB interaction (Bian et al., 2014; Sharma et al., 2014). Furthermore, dimerisation has been implicated to enhance FFAT-VAPB interaction (Kaiser et al., 2005; Mikitova and Levine, 2012).

In summary, we suggest that the negatively charged phosphate groups support the overall acidic environment by increasing affinity for the electropositive face of the VAP-MSP domain, thus facilitating FFAT-MSP interaction (Fig. 2B, Fig. 8). An alternative explanation may be that phosphorylation induces structural rather than electrostatic changes, which enhance VAP binding. An extended acidic tract may be particularly important for VAP binding of proteins with "weaker" FFAT motifs. The prediction of non-canonical FFAT motifs is problematic [e.g. the ACBD4 FFAT-like motif has a FFAT score of 3.5, which is outside the generally-used cut-off of 2.5 (Murphy and Levine, 2016; Slee and Levine, 2019)]. It is possible that serine/threonine residues within and upstream of the acidic tract contribute more prominently to VAP affinity than is currently factored into the scoring algorithm 
(Murphy and Levine, 2016). Such residues may therefore need to be considered so that proteins with a lower FFAT score are not omitted.

Substitution of the serine residue at position 5 of the FFAT core to phosphomimetic glutamic acid in both ACBD4 and ACBD5 abolished their binding to VAPB completely (Fig. S3B, Fig. 3A). A previous study, in which peptides of human proteins were expressed in yeast, reported that glutamic acid at this position in the FFAT core of human AKAP220 completely inhibited ER-localisation, suggesting a loss of binding to the yeast VAP homolog Scs2p (Mikitova and Levine, 2012). Furthermore, we show that the serine at position 5 of the ACBD5 FFAT core (S269) can be phosphorylated (Fig. 3C, D) and that this phosphorylated population is only detectable in the fraction not immunoprecipitated by VAPB, indicating that FFAT phosphorylation at position 5 blocks VAP interaction. (Fig. 3E). The canonical FFAT motif has an alanine residue at this position ( ${ }^{1}$ EFFDA-E $\left.{ }^{7}\right)$, which binds VAP in a small hydrophobic pocket (Furuita et al., 2010; Kaiser et al., 2005) (Fig. 2B). Steric hindrance likely excludes glutamic acid and phosphorylated serine from this pocket, and thereby binding of the peptide/protein to VAP. At first glance, phosphorylation within the FFAT-like motif that inhibits VAPB binding appears to be in contrast to our observation that phosphatase treatment/dephosphorylation of ACBD5 inhibits VAPB binding (see Fig. 1). However, phosphorylation of residues within the acidic tract (outside the core region) is likely required to initiate a more transient ACBD5-VAPB interaction prior to interaction of the FFAT core with the MSP hydrophobic pocket (Fig. 2). We speculate that phosphorylation of the serine residue within the FFAT core might represent an additional regulatory mechanism to modulate ACBD5-VAPB interaction. Importantly, phosphorylation of the serine residue at position 5 may also negatively regulate VAP interaction of other proteins with a FFAT motif; numerous FFAT motifcontaining proteins possess a serine or threonine at this position (Fig. S5) (Murphy and Levine, 2016).

Phosphorylation upstream of the FFAT motif may also alter the phosphorylation status of the critical serine residue in the core of the ACBD4/5 FFAT-like motif (position 5). ACBD5 with nonphosphorylatable residues in the acidic tract showed reduced levels of pS269 (Fig. 6C). 'Crosstalk' between (non)phosphorylated residues/regions, in and outside the FFAT region in the regulation of the FFAT-VAP interaction and protein function has been suggested previously (Goto et al., 2012; Kumagai et al., 2014).

Interestingly, recent studies revealed that phosphorylation of serine and threonine residues at position 4 of the FFAT core strongly increases the affinity of unconventional motifs to VAP (Di Mattia et al., 2020; Guillén-Samander et al., 2021), showing that phosphorylation of the core can affect the FFAT-VAP binding in paradoxical ways, dependent on the position of the residue. The canonical FFAT motif possesses aspartic acid (D) at position 4, which resembles phosphorylated serine/threonine to enable VAP interaction, while the canonical FFAT motif possesses alanine (A) at position 5 , which when replaced by phosphorylated serine/threonine inhibits VAP interaction. SNX2 and CALCOCO1, two confirmed VAP interactors (Dong et al., 2016; Nthiga et al., 2020), have serine/threonine residues at both position 4 and 5 of their FFAT motif (Fig. S5), suggesting that VAP binding of these proteins could be tightly regulated by two opposing phosphorylation mechanisms.

We demonstrate that GSK3 $\beta$ is in a complex with ACBD5 and VAPB (Fig. 6) and that the kinase regulates the binding of ACBD5 to VAPB; expression of GSK3 $\beta$ decreased the ACBD5-VAPB interaction, whereas inhibition of GSK3 $\beta$ increased it (Fig. 5). Inhibition of the ACBD5-VAPB binding by GSK3 $\beta$ is 
dependent on the serine in the FFAT core of ACBD5 (S269) (Fig. 7A). Furthermore, we show that GSK3 $\beta$ can directly phosphorylate ACBD5 at this residue in vitro (Fig. 7B). Hence, we show that expression of GSK3 $\beta$ alters peroxisome-ER associations (Fig. 7C, D, Fig. S4C). Overall, our data indicates that GSK3 $\beta$ negatively regulates the ACBD5-VAPB interaction by phosphorylating ACBD5 at S269. However, GSK3 $\beta$ acts on a large number of substrates, and its role in ACBD5-VAPB regulation could be via multiple levels (Frame and Cohen, 2001). Phosphorylation of VAPB was increased upon AKT inhibition, an upstream regulator of GSK3 $\beta$ (Wiechmann et al., 2021). Other protein kinases and phosphatases directly acting on ACBD5 await identification.

The previous studies showing that GSK3 $\beta$ activity regulates the PTPIP51-VAPB interaction, and thus mitochondria-ER associations, also linked the regulation of GSK3 $\beta$ activity to TDP-43 and FUS, two proteins associated with amyotrophic lateral sclerosis (ALS) and fronto-temporal dementia (FTD) (Stoica et al., 2014, 2016). This suggests that alterations of both mitochondrial-ER and peroxisome-ER contacts might be a feature of TDP-43/FUS-induced pathology. Recently, lipid alterations in the frontal cortex of patients with ALS/FTD-TDP-43 proteinopathy have been related to peroxisome impairment (Andrés-Benito et al., 2021). Several PE- and PC-plasmalogens were found to be down-regulated, similar to decreased levels reported in ACBD5 deficient patient cell lines and ACBD5 depleted cells (Hua et al., 2017; Herzog et al., 2018). As plasmalogen biosynthesis, which is initiated in peroxisomes and completed in the ER, requires peroxisome-ER cooperation, altered peroxisome-ER contacts could contribute to TDP-43-induced pathology. Aberrant activity of GSK3 $\beta$ has also been linked to peroxisomal disorders; GSK3 $\beta$ activity was increased in the nervous system of mouse models for adrenoleukodystrophy (ALD) and rhizomelic chondrodysplasia punctata (RCDP) and in patient fibroblasts (da Silva et al., 2014; Ranea-Robles et al., 2018). Whether peroxisome-ER contacts are impaired under those conditions awaits further research.

Peroxisome-ER contact sites mediated by ACBD5-VAPB have been implicated in the regulation of peroxisome motility and positioning, membrane expansion and biogenesis of peroxisomes, as well as metabolic cooperation (e.g. in plasmalogen synthesis) (Costello et al., 2017b; Hua et al., 2017). Switching peroxisome-ER tethering "ON" and "OFF" would allow regulation of these processes under different physiological conditions. For example, the control of peroxisome motility and positioning may be critical for cell division, in which peroxisome inheritance plays a role in normal cell mitosis and differentiation (Asare et al., 2017). The physiological function(s) of ACBD4-VAPB mediated peroxisome-ER contacts remain to be elucidated; they may differ from those of ACBD5-VAPB, as ACBD4 responds differently to (de)phosphorylation.

Many peroxisomal proteins, involved in various processes such as peroxisome biogenesis and protein import, are phosphorylated according to phospho-proteomics studies, but the biological function of this phosphorylation remains largely unknown (Oeljeklaus et al., 2016). Phosphorylation of human PEX5, the shuttling import receptor for peroxisomal matrix proteins, has been shown to be implicated in pexophagy in response to ROS (Zhang et al., 2015). Phosphorylation of mammalian PEX14, a membrane protein that is part of the peroxisomal import machinery, suppressed the import of catalase into peroxisomes under oxidative stress conditions and in mitotic cells (Okumoto et al., 2020; Yamashita et al., 2020). Additionally, Pex14p phosphorylation in the yeast $S$. cerevisiae controls the import of Cit2p, the peroxisomal isoform of citrate synthase (Schummer et al., 2020). Other studies in yeast also provide insight into the physiological role of phosphorylation of peroxisomal proteins. In $S$. 
cerevisiae and P. pastoris, the peroxisome biogenesis factor Pex11p is activated by site-specific phosphorylation (Knoblach and Rachubinski, 2010; Joshi et al., 2012), although this appears to be different in H. polymorpha (Thomas et al., 2015). In addition, pexophagy requires the phosphorylation of pexophagy receptor Atg30 in P. pastoris (Burnett et al., 2015). Our novel findings on the regulation of the ACBD5-VAPB tether, and subsequent peroxisome-ER membrane contacts, represent another example for a physiological role of phosphorylation of peroxisomal membrane proteins in mammals. 


\section{MATERIALS AND METHODS}

\section{Plasmids and antibodies}

See Table S1 for details of plasmids used in this study, Table S2 for plasmids generated in this study, Table S3 for gene synthesis of ACBD4 with ACBD5 FFAT-like motif region, Table S4 for gene synthesis of ACBD5 codon optimised for E. coli, and Table S5 for details of primers used. Site-directed mutagenesis to generate point mutations was done using the QuikChange Site-Directed Mutagenesis kit (Agilent Technologies). All constructs produced were confirmed by sequencing (Eurofins Genomics).

The polyclonal rabbit phospho-ACBD5 Ser269 antibody ( $\alpha$-ACBD5 pS269) was produced by Eurogentec (Seraing, Belgium). The antibody was raised against peptide ${ }^{264} \mathrm{EVYCDSMEQFGQE}^{276}$ including a phospho-Ser269. Phospho-specific and non-phospho-specific ( $\alpha$-ACBD5 control) antibodies targeting the peptide were purified from serum by double affinity purification. Details on antibodies used in this study can be found in Table $\mathbf{S 6}$.

\section{Cell culture, transfection and drug treatment}

COS-7 (African green monkey kidney cells, CRL-1651; ATCC), HEK293T (Human embryonic kidney 293T cells; ECACC), ACBD5 KO HeLa cells (generated by J. Koster, University of Amsterdam, Netherlands) (Ferdinandusse et al., 2017) and MFF-deficient fibroblasts (provided by F.S. Alkuraya, King Faisal Specialist Hospital and Research Center, Riyadh, Saudi Arabia) (Shamseldin et al., 2012; Costello et al., 2017b) were cultured in Dulbecco's Modified Eagle's Medium (DMEM), high glucose (4.5 g/L) supplemented with $10 \%$ fetal bovine serum (FBS), $100 \mathrm{U} / \mathrm{ml}$ penicillin and $100 \mu \mathrm{g} / \mathrm{ml}$ streptomycin (all from Life Technologies) at $37^{\circ} \mathrm{C}$ with $5 \% \mathrm{CO}_{2}$ and $95 \%$ humidity. COS-7 and HEK293T cells were transfected using diethylaminoethyl (DEAE)-dextran (Sigma-Aldrich) as described (Bonekamp et al., 2010) and HeLa cells with Lipofectamine 3000 (Invitrogen). HEK293T cells were seeded in dishes coated with Collagen R solution $0.4 \%$ (1:10, Serva). Transfection of fibroblasts was performed using the Neon Transfection System (Thermo Fisher Scientific) as previously described (Costello et al., 2017b). Cells were assayed for immunofluorescence or immunoblotting and immunoprecipitation experiments 24 or $48 \mathrm{~h}$ after transfection, respectively. To inhibit GSK3 $\beta$ activity, cells were treated with $10 \mu \mathrm{M}$ CHIR99021 (Sigma-Aldrich; $5 \mathrm{mM}$ stock in dimethyl sulphoxide [DMSO]) and incubated for 16 hours before immunoprecipitation. Control cells were incubated with the same volume of DMSO.

\section{Immunofluorescence and microscopy of cultured cells}

Cells grown on glass coverslips were fixed with 4\% paraformaldehyde (PFA; in PBS, pH 7.4) for 20 min, permeabilised with $0.2 \%$ Triton X-100 for $10 \mathrm{~min}$, and blocked with $1 \%$ BSA for 10 min. Blocked cells were sequentially incubated with primary and secondary antibodies (Table S6) for $1 \mathrm{~h}$ in a humid chamber at room temperature. Coverslips were washed with $\mathrm{ddH} 2 \mathrm{O}$ to remove PBS and mounted on glass slides using Mowiol medium. Cell imaging was performed using an Olympus IX81 microscope equipped with an UPlanSApo 100x/1.40 oil objective (Olympus Optical). Digital images were taken with a CoolSNAP HQ2 CCD camera and adjusted for contrast and brightness using MetaMorph 7 (Molecular Devices).

\section{Electron microscopy and spatial stereology}

Electron microscopy was performed as previously described (Costello et al., 2017b). In brief, monolayers of cells were fixed for a total of $1 \mathrm{~h}$ in $0.5 \%$ glutaraldehyde in $0.2 \mathrm{M} \mathrm{PIPES}$ buffer $(\mathrm{pH} 7.2$ ), 
and post-fixed in $1 \%$ osmium tetroxide (reduced with $1.5 \% \mathrm{w} / \mathrm{v}$ potassium ferrocyanide) in cacodylate buffer for $1 \mathrm{~h}$. After washing in deionised water the cells were dehydrated in a graded ethanol series before embedding in Durcupan resin (Sigma Aldrich). $60 \mathrm{~nm}$ ultra-thin sections were collected on pioloform-coated 100 mesh copper EM grids (Agar Scientific) and contrasted with lead citrate prior to imaging using a JEOL JEM 1400 transmission electron microscope operated at $120 \mathrm{kV}$. Images were taken with a digital camera (ES 1000W CCD, Gatan). Quantification of peroxisome-ER contacts was performed as previously (Costello et al., 2017b). In brief, peroxisomes were sampled ( $n=36-55$, mean $=44 \pm 1$ (Fig. 4) or $n=106-116$, mean $=112 \pm 1.46$ (Fig. 7) peroxisomes per experimental grid) by scanning the EM grids systematic uniform random. To estimate the mean fraction of total peroxisome membrane surface in direct contact with the $E R$, a stereological approach by line intersection counting was used. Intersections were classified as direct membrane contact (defined as "attachment") if there was $<15 \mathrm{~nm}$ distance between peroxisome and ER membranes.

\section{Protein extraction and phosphatase treatment}

Transfected cells and controls were washed in PBS, and lysed in ice-cold lysis buffer (50-100 mM Tris$\mathrm{HCL} \mathrm{pH} \mathrm{7.4,} 150 \mathrm{mM} \mathrm{NaCl}$ 2mM DTT, 1\% Triton X-100, and mini protease inhibitor cocktail (Roche), with or without phosphatase inhibitor cocktail (Roche)). Insolubilized material was pelleted by centrifugation at $15,000 \times \mathrm{g}$. Clarified lysates were incubated with $0.9 \mathrm{mM} \mathrm{MnCl} 2$ and $7 \mathrm{U} / \mu$ l lysate lambda protein phosphatase ( $\lambda$ PP; New England BioLabs), or $0.9 \mathrm{mM} \mathrm{MnCl}_{2}$ and $\mathrm{H}_{2} \mathrm{O}$ as control, for 1 $\mathrm{h}$ at $30^{\circ} \mathrm{C}$. The total protein concentration of the lysate was determined by a Bradford protein assay (Bio-Rad). Reactions were stopped and proteins were denatured in Laemmli buffer for $10 \mathrm{~min}$ at $95^{\circ} \mathrm{C}$.

\section{SDS-PAGE and immunoblotting}

Proteins were separated on $10 \%$ or $12.5 \%$ conventional SDS-PAGE gels, and subsequently transferred to nitrocellulose membranes (Amersham Bioscience) using a semi-dry apparatus (Trans-Blot SD, BioRad). Phos-tag, in complex with a divalent cation, binds reversibly to phosphate groups, separating phosphorylated and non-phosphorylated forms of proteins when added to polyacrylamide gels (Kinoshita et al., 2006). For Phos-tag SDS-PAGE, $50 \mu \mathrm{M}$ Phos-tag acrylamide (Wako Chemicals, purchased from NARD Institute Itd.) and $100 \mu \mathrm{M} \mathrm{MnCl}_{2}$ were added to the resolving gel solution of $8 \%$ SDS-PAGE gels before polymerization. To improve electrotransfer of ACBD5, a two-layer Phos-tag SDSPAGE method was used (Longoni et al., 2015); the Phos-tag is only present in the upper layer of the resolving gel. For two-layer Phos-tag SDS-PAGE, the same amounts of Phos-tag and $\mathrm{MnCl}_{2}$ were added to $6 \%$ acrylamide resolving gel solution. One volume of the Phos-tag resolving gel solution was drawn into a serological pipet. Using the same pipet, three volumes of normal $6 \%$ acrylamide resolving gel solution was drawn up. Ejection of the gel solution between the glass plates resulted in a gel with only the Phos-tag ligand in the top of the gel. Conventional SDS-PAGE was performed in parallel, including $\mathrm{MnCl}_{2}$ in the corresponding layers as control. After protein separation at a constant voltage of $75 \mathrm{~V}$ for $3 \mathrm{~h}$, the Phos-tag SDS PAGE gel was incubated in transfer buffer containing $10 \mathrm{mM}$ EDTA to remove $\mathrm{Mn}^{2+}$. Proteins were transferred to activated PVDF membranes (GE Healthcare) using the semi-dry method for $1 \mathrm{~h}$ at $24 \mathrm{~V}$. Membranes were blocked in 5\% dry milk (Marvel) in Tris-buffered saline with Tween-20 (TBS-T), and incubated with primary antibodies (Table S6), followed by incubation with horseradish peroxidase-conjugated secondary antibodies (Table S6) and detected with enhanced chemiluminescence reagents (Amersham Bioscience) using Amersham hyperfilm (GE Healthcare) or the G:Box Chemi (Syngene). For antibody reprobing, membranes were incubated 2-3 times for 10-15 min in a mild membrane stripping buffer ( $1.5 \% \mathrm{w} / \mathrm{v}$ glycine, $0.1 \% \mathrm{w} / \mathrm{v}$ SDS, $1 \% \mathrm{v} / \mathrm{v}$ Tween-20, pH 2.2 
$(\mathrm{HCl}))$. Following this, the membranes were washed in TBS-T and blocked in $5 \%$ dry milk in TBST-T prior to antibody incubation.

\section{Immunoprecipitation (IP) for in vitro binding assays}

For in vitro binding assays (Fig. 1B), GST-VAPB was expressed in BL21 Rosetta (DE3) cells (EMD Millipore) induced with $1 \mathrm{mM}$ IPTG for $4 \mathrm{~h}$. Cells were centrifuged at 5,000x $\mathrm{g}$ for $10 \mathrm{~min}$ and pellets resuspended in ice-cold Escherichia coli lysis buffer (50 mM Tris- $\mathrm{HCl}, \mathrm{pH} 7.5,300 \mathrm{mM} \mathrm{NaCl}, 0.1 \% \mathrm{NP}-$ 40, $1 \mathrm{mM}$ PMSF, and protease inhibitor cocktail). Cells were disrupted by sonication, and insoluble material was removed by centrifugation at $15,000 \times \mathrm{g}$. The supernatant was incubated with GST-TRAP agarose beads (ChromoTek) for $2 \mathrm{~h}$ at $4^{\circ} \mathrm{C}$. COS-7 cell lysates from FLAG-ACBD4/5 (mFFAT) expressing cells were treated for $1 \mathrm{~h}$ with $\lambda$ PP (New England BioLabs) as described above, and subsequently incubated with the GST-VABP-bound beads for $30 \mathrm{~min}$. Beads were then washed extensively with wash buffer (50mM Tris-HCL pH 7.4, $150 \mathrm{mM} \mathrm{NaCl}, 2 \mathrm{mM}$ DTT, $1 \%$ Triton X-100) in a rotating shaker at $4^{\circ} \mathrm{C}$ and by centrifugation at $2,500 \mathrm{x}$ g. Proteins were eluted with Laemmli buffer for 10 minutes at $95^{\circ} \mathrm{C}$. Immunoprecipitates and total lysates were analysed by Western immunoblotting.

\section{IP for other binding assays}

For binding assays (Fig. 1E, S1A), COS-7 cell lysates from FLAG-ACBD4(wACBD5_FFAT)/5 (mFFAT) expressing cells were treated for $1 \mathrm{~h}$ with $\lambda$ PP (New England BioLabs) as described above. Subsequently, DTT and Triton X-100 concentrations were adjusted to $0.4 \mathrm{mM}$ and $0.2 \%$, respectively, by using dilution buffer (50-100 mM Tris-HCL pH 7.4, $150 \mathrm{mM} \mathrm{NaCl}$ ). The samples were incubated with anti-FLAG M2 affinity gel (Sigma) at $4^{\circ} \mathrm{C}$ for $1 \mathrm{~h}$, after which the gel was repeatedly washed with dilution buffer in a rotating shaker at $4^{\circ} \mathrm{C}$ and by centrifugation at 5,000x g. Proteins were competitively eluted using 3X FLAG peptide (Sigma; in $10 \mathrm{mM}$ Tris HCl, $150 \mathrm{mM} \mathrm{NaCl}, \mathrm{pH} 7.4$ (TBS)). Immunoprecipitates and total lysates were analysed by Western immunoblotting.

For quantification of ACBD5-VAPB binding (Fig. 1C), Myc-ACBD5 was expressed in COS-7 cells and cells lysed, compatible with $\lambda$ PP-treatment, as described above. Insolubilized material was removed by centrifugation at $100,000 \mathrm{x}$ for $20 \mathrm{~min}$ at $4^{\circ} \mathrm{C}$. Clarified lysates were treated for $1 \mathrm{~h}$ with $\lambda \mathrm{PP}$ (New England BioLabs) as described above. Subsequently, DTT and Triton X-100 concentrations were adjusted to $0.66 \mathrm{mM}$ and $0.33 \%$, respectively, by using dilution buffer (50 mM Tris-HCL pH 7.4, 150 $\mathrm{mM} \mathrm{NaCl}$ ). Lysates were then mixed with Myc-TRAP magnetic agarose beads (ChromoTek) and incubated for $1 \mathrm{~h}$ at $4^{\circ} \mathrm{C}$ on a rotating wheel. Beads were washed extensively with dilution buffer and bound proteins were eluted with Laemmli buffer for Western immunoblotting.

\section{IP of phospho-mutants and GSK3 $\beta$ experiments}

For immunoprecipitation of FLAG-ACBD4 phospho-mutants (Fig. S3A, B) or FLAG-ACBD5/VAPB (Fig. $5 B$, Fig. 6), the constructs mentioned in the experiments were expressed in COS-7 cells. Cells were washed in PBS, and lysed in ice-cold lysis buffer (50 mM Tris- $\mathrm{HCl}$ pH7.4, $150 \mathrm{mM} \mathrm{NaCl}, 1 \mathrm{mM}$ EDTA, $1 \%$ Triton X-100, mini protease inhibitor cocktail (Roche), and phosphatase inhibitor cocktail (Roche)). Insolubilized material was pelleted by centrifugation at 15,000x g. The supernatant was incubated with anti-FLAG M2 affinity gel (Sigma) and further processed as described above (beads were washed with FLAG wash buffer: $50 \mathrm{mM}$ Tris-HCl pH7.4, $150 \mathrm{mM} \mathrm{NaCl}, 1 \mathrm{mM}$ EDTA, 1\% Triton X-100).

For immunoprecipitation of Myc-ACBD5 phospho-mutants (Fig. 2C, 3A, D, 7A, S1B) or Myc-VAPB (Fig. 5A, S4B), the constructs mentioned in the experiments were expressed in COS-7 cells. After $48 \mathrm{~h}$ cells were washed in PBS, and lysed in ice-cold lysis buffer $(10 \mathrm{mM}$ Tris-HCL pH 7.4, $150 \mathrm{mM} \mathrm{NaCl}, 0.5 \mathrm{mM}$ 
EDTA, $0.5 \%$ NP-40, mini protease inhibitor cocktail (Roche), and phosphatase inhibitor cocktail (Roche)). Insolubilized material was pelleted by centrifugation at $15,000 \mathrm{x}$. The supernatant was diluted (1:2) with dilution buffer (10 mM Tris-HCL pH 7.4, $150 \mathrm{mM} \mathrm{NaCl}, 0.5 \mathrm{mM}$ EDTA) and mixed with Myc-TRAP (ChromoTek) magnetic agarose beads and incubated for $1 \mathrm{~h}$ at $4^{\circ} \mathrm{C}$. Beads were subsequently extensively washed with dilution buffer in a rotating shaker at $4^{\circ} \mathrm{C}$. Proteins were eluted with Laemmli buffer for $10 \mathrm{~min}$ at $95^{\circ} \mathrm{C}$. Immunoprecipitates and total lysates were analysed by Western immunoblotting. For immunoprecipitation with phosphatase treatment (Fig. 2D, E, 3C), clarified lysates were treated for $1 \mathrm{~h}$ with $\lambda$ PP (New England BioLabs) as described above. DTT and Triton X-100 concentrations were adjusted to $0.4 \mathrm{mM}$ and $0.2 \%$, respectively, by using dilution buffer (100 mM Tris-HCL pH 7.4, $150 \mathrm{mM} \mathrm{NaCl}$ ), prior to beads incubation. The samples were further processed as described above.

\section{IP of FLAG-ACBD5 binding to Myc-VAPB}

To assess the binding of FLAG-ACBD5 pS269 to Myc-VAPB (Fig. 3E), Myc-VAPB and FLAG-ACBD5 were expressed separately in COS-7 cells. Myc-VAPB was immunoprecipitated as described above. Beads were extensively washed with Myc wash buffer (10 mM Tris-HCL pH 7.4, $150 \mathrm{mM} \mathrm{NaCl}, 0.5 \mathrm{mM}$ EDTA, $0.05 \%$ NP-40). FLAG-ACBD5 was immunoprecipitated as described above. The washes with FLAG wash buffer were followed by a wash in Myc wash buffer. Proteins were competitively eluted using 3X FLAG peptide (Sigma; in Myc wash buffer). The eluted FLAG-ACBD5 was incubated with the Myc-VAPBbound beads for $1 \mathrm{~h}$ at $4^{\circ} \mathrm{C}$. The supernatant was transferred to a new tube and the beads were subsequently extensively washed with Myc wash buffer in a rotating shaker at $4^{\circ} \mathrm{C}$. Proteins were eluted with Laemmli buffer for $10 \mathrm{~min}$ at $95^{\circ} \mathrm{C}$. Immunoprecipitates and total lysates were analysed by Western immunoblotting.

\section{In vitro kinase assay}

His-ACBD5 was expressed in BL21 Rosetta (DE3) cells (EMD Millipore) induced with $1 \mathrm{mM}$ IPTG overnight at $18^{\circ} \mathrm{C}$. Cells were centrifuged at 5,000x g for $5 \mathrm{~min}$ and pellets re-suspended in ice-cold lysis buffer ( $50 \mathrm{mM}$ Tris- $\mathrm{HCl}, \mathrm{pH}$ 7.4, $300 \mathrm{mM} \mathrm{NaCl}, 10 \mathrm{mM}$ Imidazole, $4 \mathrm{mM}$ DTT, 1\% Triton X-100 and protease inhibitor cocktail (Roche)). Cells were disrupted by sonication, and insoluble material was removed by centrifugation at $13,000 \times \mathrm{g}$. The supernatant was incubated with HisPur ${ }^{\mathrm{TM}}$ Ni-NTA agarose beads (Thermo Fisher) for $1 \mathrm{~h}$ at $4^{\circ} \mathrm{C}$. Beads were washed extensively in wash buffer ( $50 \mathrm{mM} \mathrm{Tris-HCl}$, pH 7.4, $300 \mathrm{mM} \mathrm{NaCl}, 25 \mathrm{mM}$ Imidazole, $4 \mathrm{mM}$ DTT and protease inhibitor cocktail (Roche)) to remove unbound protein. Purified His-ACBD5 was eluted from the beads by incubating with elution buffer (50 $\mathrm{mM}$ Tris- $\mathrm{HCl}, \mathrm{pH}$ 7.4, $300 \mathrm{mM} \mathrm{NaCl}, 250 \mathrm{mM}$ Imidazole, $4 \mathrm{mM}$ DTT) for $15 \mathrm{~min}$ at room temperature. His-ACBD5 concentration was adjusted to $10 \mathrm{ng} / \mu \mathrm{l}$ by diluting in kinase reaction buffer ( $10 \mathrm{mM}$ Tris$\mathrm{HCl}, \mathrm{pH}$ 7.4, $50 \mathrm{mM} \mathrm{NaCl}, 5 \mathrm{mM} \mathrm{MgCl} 2,0.5 \mathrm{mM}$ EDTA and 0.05\% NP-40). For in vitro kinase assays (Fig. 7B), reaction mixes were prepared using $2.5 \mu \mathrm{g}$ recombinant His-ACBD5 with or without the addition of $0.3 \mathrm{mM}$ ice-cold ATP (Thermo Fisher) and $0.1 \mathrm{ug}$ GST-GSK3 $\beta$ (Abcam). Reactions were incubated at $37^{\circ} \mathrm{C}$ for $30 \mathrm{~min}$. Samples were prepared with Laemmli buffer and then analysed by Western immunoblotting.

\section{Mass spectrometry (MS)}

FLAG-ACBD5 expressed in COS-7 cells was immunoprecipitated using anti-FLAG M2 affinity gel (Sigma) as described above. Subsequently, beads were washed twice in ammonium bicarbonate $(50 \mathrm{mM})$ and cysteine residues were reduced with $5 \mathrm{mM}$ tris(2-carboxyethyl)phosphine (20 min, $800 \mathrm{rpm}, 37^{\circ} \mathrm{C}$ ) and 
alkylated with $50 \mathrm{mM}$ 2-chloroacetamide $\left(20 \mathrm{~min}, 800 \mathrm{rpm}, 25^{\circ} \mathrm{C}\right)$. Proteins were digested on-bead either with sequencing-grade trypsin (1:50) (Promega, Walldorf, Germany) for $4 \mathrm{~h}$ at $800 \mathrm{rpm}$ and $42^{\circ} \mathrm{C}$ or thermolysine (1:50) (Promega, Walldorf, Germany) for $2 \mathrm{~h}$ at $800 \mathrm{rpm}$ and $60^{\circ} \mathrm{C}$. Peptides were acidified using TFA at a final concentration of $1 \%$ and phosphopeptide enrichment was performed using titanium dioxide $\left(\mathrm{TiO}_{2}\right)$ as described previously with slight modifications (Humphrey et al., 2018). Before incubation with proteolytic peptide samples, $\mathrm{TiO}_{2}$ beads were washed using elution and wash buffer. $\mathrm{C} 8$ stage tips were pre-equilibrated with methanol and wash buffer. For mass spectrometric analysis, enriched and non-enriched peptide samples were desalted as described before (Rappsilber et al., 2003).

Reversed-phase liquid chromatography-mass spectrometry (LC-MS) was performed using the UltiMate $^{\mathrm{TM}} 3000$ RSLCnano system (Dionex LC Packings/Thermo Fisher Scientific, Dreieich, Germany) coupled online to a $Q$ Exactive Plus (Thermo Fisher Scientific, Bremen, Germany) instrument. The UHPLC system was equipped with two pre-columns (nanoEase ${ }^{\mathrm{TM}} \mathrm{M} / \mathrm{Z}$ Symmetry C18, $100 \AA, 5 \mu \mathrm{m}$, Waters or $\mu \mathrm{PAC}^{\mathrm{TM}}$ trapping column, PharmaFluidics) and a corresponding analytical column $(25 \mathrm{~cm}$ nanoEase $^{\mathrm{TM}} \mathrm{M} / \mathrm{Z}$ HSS C18 T3 column, Waters or $50 \mathrm{~cm} \mu \mathrm{PAC}^{\mathrm{TM}}$ column, PharmaFluidics). The MS instrument was externally calibrated using standard compounds and equipped with a nanoelectrospray ion source and a fused silica emitter (New Objectives). For MS analysis, dried peptides were resolved in $15 \mu \mathrm{l} 0.1 \%$ TFA and analysed with an $1 \mathrm{~h} \mathrm{LC}$ method. Gradients were applied using a binary solvent systems of $0.1 \% \mathrm{FA}\left(\mathrm{v} / \mathrm{v}\right.$, solvent $A,{ }^{\prime} \mathrm{A}$ ') and $0.1 \% \mathrm{FA} / 86 \%$ acetonitrile $(\mathrm{v} / \mathrm{v}$, solvent $B$, ' $B$ '). For nanoEase column setup, a gradient from $4 \%$ B to $42 \%$ B in $30 \mathrm{~min}$ and to $95 \%$ B in 3 min was performed, followed by a re-equilibration with $4 \%$ B for 16 min. $\mu$ PAC columns were used with a gradient from 1-24\% B in $22 \mathrm{~min}$, followed by an increase to $42 \% \mathrm{~B}$ in $11 \mathrm{~min}$ and to $95 \%$ B in 6 min. Re-equilibration was performed with $1 \% B$ for $16 \mathrm{~min}$. Full scans were acquired for a mass range of $375-1,700 \mathrm{~m} / \mathrm{z}$ with a resolution of 70,000 at $200 \mathrm{~m} / \mathrm{z}$. The automatic gain control (AGC) was set to $3 \mathrm{e}^{6}$ ions with a max. ion time (IT) of $60 \mathrm{~ms}$. MS/MS analyses of multiply charged peptide ions were generally performed using a top12 method and higher-energy collisional dissociation (HCD) with an energy of 28 and an exclusion time of $45 \mathrm{~s}$. The resolution for MS/MS scans was 35,000 and the AGC $1 \mathrm{e} 5$ with a max. IT of $120 \mathrm{~ms}$.

\section{Statistical analysis}

Protein sequence alignment was performed by Clustal Omega (1.2.4) Multiple Sequence Alignment (Madeira et al., 2019). Immunoblot signals were quantified using ImageJ or GeneTools (Syngene) for film and CCD images, respectively. Two-tailed unpaired t-tests were used for statistical comparisons between 2 groups. For experiments containing more groups, one-way ANOVA with Dunnett's post hoc test was used to determine statistical differences against a control mean, or one-way ANOVA with Tukey's post hoc test was used to determine statistical difference between the mean of all possible pairs. Statistical analyses were performed on GraphPad Prism (v8.1.2). Data are presented as mean \pm SEM. ${ }^{*} p<0.05, * * p<0.01, * * * p<0.001, * * * *<<0.0001$.

\section{Bioinformatics}

Peak lists obtained from MS/MS spectra were identified using Mascot version 2.6.1 [PMID 10612281] and MS Amanda version 2.0.0.9695 [PMID 24909410]. The search was conducted using SearchGUI version [3.3.17] [PMID 21337703]. Protein identification was conducted against a concatenated target/decoy [PMID 20013364] version of the Homo sapiens complement of the UniProtKB (version of 04/2019; 95,916 target sequences). The decoy sequences were created by reversing the target 
sequences in SearchGUI. The identification settings were as follows: trypsin, specific, with a maximum of 4 missed cleavages; thermolysin, unspecific; both with 5 ppm as MS1 and 0.5 Da as MS2 tolerances. Fixed modifications were set to: carbamidomethylation of C; variable modifications to: acetylation of protein $\mathrm{N}$-term, phosphorylation of $\mathrm{S}$ and $\mathrm{T}$, oxidation of $\mathrm{M}$. All algorithm-specific settings are listed in the Certificate of Analysis available in the supplementary information. Peptides and proteins were inferred from the spectrum identification results using PeptideShaker version 1.16.44 [PMID 25574629]. Peptide Spectrum Matches (PSMs), peptides and proteins were validated at a $1 \%$ False Discovery Rate (FDR) estimated using the decoy hit distribution. Post-translational modification localizations were scored using the D-score [PMID 23307401] and the phosphoRS score [PMID 22073976] with a threshold of 95 as implemented in the compomics-utilities package [PMID 21385435]. A phosphoRS score $>95$ was considered as a confident site localization.

\section{Data availability}

All raw data and original Mascot result files have been deposited to the ProteomeXchange Consortium (http://proteomecentral.proteomexchange.org) via the PRIDE partner repository (http://www.ebi.ac.uk/pride/archive/login) (Perez-Riverol et al., 2019) with the dataset identifier PXD018005.

\section{ONLINE SUPPLEMENTAL MATERIAL}

Fig. S1 provides additional evidence to support the data shown in Fig. 1 that the ACBD5-VAPB binding is sensitive to phosphatase treatment. Fig. S2 gives an overview of the potential phosphorylation sites involved in the ACBD4/5-VAPB interaction explored in this study. Fig. S3 provides evidence that ACBD4 phosphomimetic mutants increase VAPB interaction and shows the subcellular localisation of ACBD4/5 mutants and their effect on peroxisomal morphology. Fig. S4 shows that expression of GSK3 $\beta$ results in increased cellular activity of the kinase, and that this affects peroxisome morphology in dMFF cells. Additionally, it explores the interaction of VAPB mMSP with ACBD5. Fig S5 shows that in addition to ACBD4 and ACBD5, other FFAT-containing proteins have a serine/threonine residue at position 5 of the FFAT core.

\section{ACKNOWLEDGEMENTS}

We thank all colleagues who provided cell lines, plasmids, and antibodies (see Tables S1-S6 for more information), T.A. Schrader, A. Correia and P. Cherek for technical assistance and the PRIDE team for data deposition to the ProteomeXchange Consortium. This work was supported by grants from the Biotechnology and Biological Sciences Research Council (BB/N01541X/1 to M. Schrader; BB/T002255/1 to M. Schrader and J.L. Costello), Medical Research Council (CiC 08135 to M. Schrader), UKRI Future Leader Fellowship Award (MR/T019409/1 to J.Costello), Royal Society Research Grant Award (RGS\R2\192378 to J. Costello), University of Exeter, and the European Union's Horizon 2020 research and innovation programme under the Marie Skłodowska-Curie grant agreement No 812968 PERICO (to M. Schrader and B. Warscheid) as well as the Deutsche Forschungsgemeinschaft (DFG, German Research Foundation) Project-ID 278002225/GRK 2202 (to B. Warscheid), FOR 1905 (PerTrans; to B. Warscheid), Project-ID 403222702 - SFB 1381 (to B. Warscheid) and under Germany's Excellence Strategy - EXC-2189 - Project-ID 390939984 (CIBSS; to B. Warscheid). S. Kors is supported by the GW4 BioMed MRC Doctoral Training Partnership (MR/N0137941/1). Work included in this study has also been performed in partial fulfilment of the doctoral theses of R. Maier at the University 
of Freiburg. The research data supporting this publication are provided within this paper, as supplementary information, or are deposited on PRIDE.

\section{AUTHOR CONTRIBUTIONS}

C. Hacker, C. Bolton, R. Maier, L. Reimann, and E.J.A. Kitchener performed experiments and analyzed data. M. Schrader, J.L. Costello, B. Warscheid, and S. Kors conceived the project, performed experiments, and analyzed data. M. Schrader, J.L. Costello, and S. Kors wrote the manuscript, and all authors contributed to methods.

\section{CONFLICT OF INTEREST}

The authors declare no competing financial interests.

\section{ABBREVATIONS}

ACBD, acyl-coenzyme A-binding domain containing protein; ER, endoplasmic reticulum; FFAT, two phenylalanines (FF) in an acidic tract; $\lambda P P, \lambda$ protein phosphatase; GSK3 $\beta$, glycogen synthase kinase-3 beta; IP, immunoprecipitation; MS, mass spectrometry; MSP, major sperm protein; VAP, vesicleassociated membrane protein (VAMP)-associated protein; VLCFA, very long-chain fatty acid. 


\section{SUPPLEMENTARY INFORMATION}

Table S1. Plasmids used in this study. Number indicates isoform.

\begin{tabular}{lll}
\hline Plasmid & Source & Vector \\
\hline FLAG-ACBD4.2 & (Costello et al., 2017c) & pCMV-Tag2B \\
FLAG-ACBD5.1 & (Costello et al., 2017b) & pCMV-Tag2B \\
FLAG-ACBD5.1 mFFAT & (Costello et al., 2017b) & pCMV-Tag2B \\
Myc-ACBD5.1 & (Costello et al., 2017b) & pCMV-Tag3B \\
GSK3ß & C. Miller, King's College London, England, UK & \\
Myc-VAPB & C. Miller, King's College London, England, UK & pCI-neo \\
GST-VAPB & (Costello et al., 2017b) & pGEX-6p2 \\
- & M. Wilmanns, EMBL Hamburg, Germany & pETM12 \\
\hline
\end{tabular}

Table S2. Plasmids generated in this study.

\begin{tabular}{|c|c|c|c|c|}
\hline Plasmid & Template & Primers & Enzymes & Vector \\
\hline \multirow[t]{4}{*}{ FLAG-ACBD4.2 mFFAT } & FLAG-ACBD4.2 & A4_mFFAT_1_Fw & & pCMV-Tag2B \\
\hline & & A4_mFFAT_1_Rv & & \\
\hline & & A4_mFFAT_2_Fw & & \\
\hline & & A4_mFFAT_2_Rv & & \\
\hline \multirow[t]{2}{*}{ FLAG-ACBD4.2 with ACBD5 FFAT } & Gene synthesis from & & EcoRV & pCMV-Tag2B \\
\hline & Eurofins (Table S3) & & Xhol & \\
\hline \multirow[t]{2}{*}{ FLAG-ACBD4.2 S166E } & FLAG-ACBD4.2 & A4_S166E_Fw & & pCMV-Tag2B \\
\hline & & A4_S166E_Rv & & \\
\hline \multirow[t]{2}{*}{ FLAG-ACBD4.2 S166A } & FLAG-ACBD4.2 & A4_S166A_Fw & & pCMV-Tag2B \\
\hline & & A4_S166A_Rv & & \\
\hline \multirow[t]{2}{*}{ FLAG -ACBD4.2 S169E } & FLAG-ACBD4.2 & A4_S169E_Fw & & pCMV-Tag2B \\
\hline & & A4_S169E_Rv & & \\
\hline \multirow[t]{2}{*}{ FLAG-ACBD4.2 S169A } & FLAG-ACBD4.2 & A4_S169A_Fw & & pCMV-Tag2B \\
\hline & & A4_S169A_Rv & & \\
\hline \multirow[t]{2}{*}{ FLAG-ACBD4.2 S166ES169E } & FLAG-ACBD4.2 S166E & A4_(S166E)S169E_Fw & & pCMV-Tag2B \\
\hline & & A4_(S166E)S169E_Rv & & \\
\hline \multirow[t]{2}{*}{ FLAG-ACBD4.2 S166AS169A } & FLAG-ACBD4.2 S166A & A4_(S166A)S169A_Fw & & pCMV-Tag2B \\
\hline & & A4_(S166A)S169A_Rv & & \\
\hline \multirow[t]{2}{*}{ FLAG-ACBD4.2 S166ES169ES171E } & FLAG-ACBD4.2 & A4_(S166ES169E)S171E_Fw & & pCMV-Tag2B \\
\hline & S166ES169E & A4_(S166ES169E)S171E_Rv & & \\
\hline \multirow[t]{2}{*}{ FLAG-ACBD4.2 S166AS169AS171A } & FLAG-ACBD4.2 & A4_(S166AS169A)S171A_Fw & & pCMV-Tag2B \\
\hline & S166AS169A & A4_(S166AS169A)S171A_Rv & & \\
\hline \multirow[t]{2}{*}{ FLAG-ACBD4.2 S183E } & FLAG-ACBD4.2 & A4_S183E_Fw & & pCMV-Tag2B \\
\hline & & A4_S183E_Rv & & \\
\hline \multirow[t]{2}{*}{ FLAG-ACBD4.2 S183A } & FLAG-ACBD4.2 & A4_S183A_Fw & & pCMV-Tag2B \\
\hline & & A4_S183A_Rv & & \\
\hline \multirow[t]{2}{*}{ FLAG-ACBD5.2 } & FLAG-ACBD5.1 & A5_iso2_Fw & EcoRV & pCMV-Tag2B \\
\hline & & A5_iso2_Rv & Xhol & \\
\hline FLAG-ACBD5.2 mFFAT & FLAG-ACBD5.1 mFFAT & A5_iso2_Fw & EcoRV & pCMV-Tag2B \\
\hline Y266K/C267K/S269R & & A5_iso2_Rv & Xhol & \\
\hline \multirow[t]{2}{*}{ FLAG-ACBD5.2 $\triangle T M D$} & FLAG-ACBD5.2 & A5_dTMD_S495X_Fw & & pCMV-Tag2B \\
\hline & & A5_dTMD_S495X_Rv & & \\
\hline \multirow[t]{2}{*}{ FLAG-ACBD5.2 S259AS261A } & FLAG-ACBD5.2 & A5_S259AS261A_Fw & & pCMV-Tag2B \\
\hline & & A5_S259AS261A_Rv & & \\
\hline \multirow[t]{2}{*}{ FLAG-ACBD5.2 S259AS261AS263A } & FLAG-ACBD5.2 & A5_(S259AS261A)S263A_Fw & & pCMV-Tag2B \\
\hline & S259AS261A & A5_(S259AS261A)S263A_Rv & & \\
\hline
\end{tabular}


His-ACBD5

Myc-ACBD5.2

Myc-ACBD5.2 mFFAT

Y266K/C267K/S269R

Myc-ACBD5.2 $\triangle \mathrm{TMD}$

Myc-ACBD5.2 S123AS124A

Myc-ACBD5.2 S136AT137A

Myc-ACBD5.2

S123AS124AS136AT137A

Myc-ACBD5.2 T252E

Myc-ACBD5.2 T252A

Myc-ACBD5.2 S259E

Myc-ACBD5.2 S259A

Myc-ACBD5.2 S261E

Myc-ACBD5.2 S261A

Myc-ACBD5.2 S263E

Myc-ACBD5.2 S263A

Myc-ACBD5.2 T258AS259A

Myc-ACBD5.2 S259AS261A

Myc-ACBD5.2 S261ES263E

Myc-ACBD5.2 S261AS263A

Myc-ACBD5.2 S259ES261ES263E

Myc-ACBD5.2 S259AS261AS263A

Myc-ACBD5.2 S269E

Myc-ACBD5.2 S269A

GSK3 $\beta$ S237E 'mFFAT'

FLAG-VAPB

FLAG-VAPB K87D

FLAG-VAPB K87DM89D mMSP
Gene synthesis from

Eurofins (Table S4)

FLAG-ACBD5.1

A5_iso2_Fw

A5_iso2_Rv

FLAG-ACBD5.1 mFFAT A5_iso2_FW

A5_iso2_Rv

Myc-ACBD5.2

A5_dTMD_S495X_Fw

A5_dTMD_S495X_Rv

Myc-ACBD5.2

Myc-ACBD5.2

Myc-ACBD5.2

S123AS124A

Myc-ACBD5.2

Myc-ACBD5.2

Myc-ACBD5.2

Myc-ACBD5.2

Myc-ACBD5.2

Myc-ACBD5.2

Myc-ACBD5.2

Myc-ACBD5.2

Myc-ACBD5.2

Myc-ACBD5.2

Myc-ACBD5.2 S263E

Myc-ACBD5.2 S263A

Myc-ACBD5.2

S261ES263E

Myc-ACBD5.2

S259AS261A

Myc-ACBD5.2

Myc-ACBD5.2

GSK3 $\beta$

Myc-VAPB

FLAG-VAPB

FLAG-VAPB K87D
A5_S123_124A_Fw

A5_S123_124A_Rv

A5_S136_T137A_Fw

A5_S136_T137A_Rv

A5_S136_T137A_Fw

A5_S136_T137A_Rv

A5_T252E_Fw

A5_T252E_Rv

A5_T252A_Fw

A5_T252A_Rv

A5_S259E_Fw

A5_S259E_Rv

A5_S259A_Fw

A5_S259A_Rv

A5_S261E_Fw

A5_S261E_Rv

A5_S261A_Fw

A5_S261A_Rv

A5_S263E_Fw

A5_S263E_Rv

A5_S263A_Fw

A5_S263A_Rv

A5_T258AS259A_Fw

A5_T258AS259A_Rv

A5_S259AS261A_Fw

A5_S259AS261A_Rv

A5_S261E(S263E)_Fw

A5_S261E(S263E)_Rv

A5_S261A(S263A)_Fw

A5_S261A(S263A)_Rv

A5_S259E(S261ES263E)_Fw

A5_S259E(S261ES263E)_Rv

A5_(S259AS261A)S263A_Fw

A5_(S259AS261A)S263A_Rv

A5_S269E_Fw

A5_S269E_Rv

A5_S269A_Fw

A5_S269A_Rv

GSK3b_S237E_Fw

GSK3b_S237E_Rv

VAPB_FLAG_FW

VAPB_FLAG_Rv

VAPB_K87D_FW

VAPB_K87D_Rv

VAPB_(K87D)M89D_FW

VAPB_(K87D)M89D_Rv

$\begin{array}{ll}\text { Ncol } & \text { pETM12 } \\ \text { Kpnl } & \\ \text { EcoRV } & \text { pCMV-Tag3B } \\ \text { Xhol } & \\ \text { EcoRV } & \text { pCMV-Tag3B } \\ \text { Xhol } & \end{array}$

pCMV-Tag3B

pCMV-Tag3B

pCMV-Tag3B

pCMV-Tag3B

pCMV-Tag3B

pCMV-Tag3B

pCMV-Tag3B

pCMV-Tag3B

pCMV-Tag3B

pCMV-Tag3B

pCMV-Tag3B

pCMV-Tag3B

pCMV-Tag3B

pCMV-Tag3B

pCMV-Tag3B

pCMV-Tag3B

pCMV-Tag3B

pCMV-Tag3B

pCMV-Tag3B

BamHI pCMV-Tag2B

EcoRV

pCMV-Tag2B

pCMV-Tag2B 


\begin{tabular}{|c|c|c|c|}
\hline \multirow[t]{2}{*}{ Myc-VAPB K87D } & Myc-VAPB & VAPB_K87D_FW & pCl-neo \\
\hline & & VAPB_K87D_Rv & \\
\hline \multirow[t]{2}{*}{ Myc-VAPB K87DM89D mMSP } & Myc-VAPB K87D & VAPB_(K87D)M89D_Fw & pCl-neo \\
\hline & & VAPB_(K87D)M89D_Rv & \\
\hline
\end{tabular}

Numbering is according to the nucleotide sequence of ACBD4 isoform 2 (UniProt identifier: Q8NC06-2) and ACBD5 isoform 2 (Q5T8D3-2).

Table S3. Sequence of ACBD4 with ACBD5 FFAT-like motif region.

\begin{tabular}{lll}
\hline Gene & Source & Sequence (5' to 3') \\
\hline ACBD4 with ACBD5 FFAT & Eurofins & GAT ATC ATG GGC ACC GAG AAA GAA AGC CCA GAG CCC GAC TGC CAG AAA CAG \\
& Genomics & TTC CAG GCT GCA GTG AGC GTC ATC CAG AAC CTG CCC AAG AAC GGT TCT TAC \\
& CGC CCC TCC TAT GAA GAG ATG CTG CGA TTC TAC AGT TAC TAC AAG CAG GCC \\
& ACC ATG GGG CCC TGC CTG GTC CCC CGG CCC GGG TTC TGG GAC CCC ATT GGA \\
& CGA TAT AAG TGG GAC GCC TGG AAC AGT CTG GGC AAG ATG AGC AGG GAG GAG \\
& GCC ATG TCT GCC TAC ATC ACT GAA ATG AAA CTG GTG GCA CAG AAG GTG ATC \\
& GAC ACA GTG CCC CTG GGT GAG GTG GCA GAG GAC ATG TTT GGT TAC TTC GAG \\
& CCC CTG TAC CAG GTG ATC CCT GAC ATG CCG AGG CCC CCA GAG ACC TTC CTG \\
& AGA AGG GTC ACA GGT TGG AAA GAG CAG GTT GTG AAT GGA GAT GTT GGG GCT \\
& GTT TCA GAG CCT CCC TGC CTC CCC AAG GAA CCG GAA GAT GTT ACA GGA ATT \\
& CAG CAT TTG ACA AGC GAT TCA GAC AGT GAA GTT TAC TGT GAT TCT ATG GAA \\
& CAA TTT GGA CAA GAA GAG TCT TTA GAC AGC TTT ACG GCA GCA TCT GGA GGA \\
& AAG CGT GAT CCC AGG AAC AGC CCC GTG CCC CCC ACA AAG AAA GAG GGG TTG \\
& CGG GGC AGC CCG CCG GGG CCC CAG GAG TTG GAC GTG TGG CTG CTG GGG ACA \\
& GTT CGA GCA CTA CAG GAG AGC ATG CAG GAG GTG CAG GCG AGG GTG CAG AGC \\
& CTG GAG AGC ATG CCC CGG CCC CCT GAG CAG AGG CCG CAG CCC AGG CCC AGT \\
& GCT CGG CCA TGG CCC CTT GGG CTC CCG GGG CCC GCG CTG CTC TTC TTC CTC \\
& CTG TGG CCC TTC GTC GTC CAG TGG CTC TTC CGA ATG TTT CGG ACC CAA AAG \\
\hline
\end{tabular}

Table S4. Condon optimised ACBD5 for expression in E. coli

\begin{tabular}{lll}
\hline Gene & Source & Sequence (5' to 3') \\
\hline ACBD5-codon-optimised & Eurofins & ATG GCT GAC ACA CGC TCT GTA CAT GAA ACG CGA TTC GAA GCC GCT GTC AAG \\
& Genomics & GTT ATA CAA AGC CTT CCA AAA AAT GGC AGT TTT CAA CCG ACC AAC GAG ATG \\
& ATG CTT AAA TTT TAC AGC TTC TAT AAG CAG GCG ACG GAG GGC CCC TGT AAG \\
& CTA TCA CGG CCC GGT TTT TGG GAT CCG ATT GGC CGC TAT AAA TGG GAC GCT \\
& TGG AGT TCG TTG GGC GAT ATG ACA AAA GAG GAA GCA ATG ATT GCA TAT GTG \\
& GAA GAA ATG AAG AAG ATT ATC GAG ACT ATG CCA ATG ACT GAG AAA GTG GAA \\
& GAG CTG CTG CGA GTG ATC GGC CCG TTC TAC GAG ATC GTT GAA GAT AAG AAA \\
& AGT GGT CGT TCA AGT GAC ATA ACA TCA GAT CTG GGG AAT GTG TTA ACT AGC \\
& ACT CCG AAC GCT AAA ACC GTC AAT GGA AAG GCT GAA TCA TCT GAT TCT GGA \\
& GCA GAA TCC GAA GAA GAG GAA GCG CAG GAA GAA GTC AAA GGC GCG GAA \\
& CAA AGT GAT AAC GAT AAA AAA ATG ATG AAA AAA TCG GCA GAC CAC AAG AAT \\
& CTT GAG GTC ATT GTA ACT AAC GGC TAT GAT AAA GAT GGA TTT GTG CAA GAT \\
& ATT CAA AAT GAC ATA CAC GCG TCT AGC TCT CTT AAC GGG CGG TCA ACC GAG \\
& GAA GTT AAA CCG ATT GAT GAA AAT TTA GGT CAG ACC GGG AAA TCC GCC GTG \\
& TGC ATC CAC CAG GAT ATT AAT GAT GAT CAT GTG GAA GAC GTC ACT GGG ATA \\
& CAA CAC CTG ACT TCC GAT TCC GAT TCC GAA GTT TAC TGC GAT AGT ATG GAA \\
& CAG TTC GGC CAA GAA GAA TCA CTG GAT AGC TTT ACA TCA AAT AAC GGA CCC \\
& TTT CAG TAT TAT CTT GGC GGC CAC AGC AGT CAG CCG ATG GAA AAC TCG GGT \\
& TTT CGG GAA GAT ATC CAA GTC CCG CCC GGG AAT GGG AAT ATT GGA AAT ATG \\
& CAA GTG GTT GCC GTG GAG GGT AAG GGT GAA GTC AAA CAT GGC GGA GAA GAT \\
& GGC CGT AAC AAT TCT GGT GCT CCA CAC CGT GAA AAG CGC GGT GGT GAG ACC \\
GAT GAA TTT TCT AAC GTG CGC CGC GGT AGG GGT CAT CGT ATG CAA CAC CTG
\end{tabular}


AGT GAA GGG ACA AAA GGC CGT CAG GTT GGA TCC GGG GGC GAC GGT GAG CGT TGG GGT AGC GAT CGC GGA TCA AGA GGA AGT TTA AAT GAG CAG ATT GCA TTG GTC CTA ATG CGT CTA CAA GAA GAT ATG CAA AAT GTA CTG CAG CGT CTT CAG AAA TTA GAA ACG CTG ACA GCC TTA CAG GCC AAA TCT TCT ACA AGC ACA TTG CAG ACC GCG CCG CAG CCG ACC TCC CAG CGA CCA TCG TGG TGG CCG TTT GAA ATG TGA

Table S5. Primers used in this study.

\begin{tabular}{|c|c|}
\hline Name & Sequence (5' to $\left.3^{\prime}\right)$ \\
\hline A4_mFFAT_1_Fw & GACCTGGACTCCGAGGTTGCCTGTGATTCCCTGGAGCAG \\
\hline A4_mFFAT_1_Rv & CTGCTCCAGGGAATCACAGGCAACCTCGGAGTCCAGGTC \\
\hline A4_mFFAT_2_Fw & GACTCCGAGGTTGCCTGTGCTTCCCTGGCGCAGCTGGAGCCTGAGCTG \\
\hline A4_mFFAT_2_Rv & CAGCTCAGGCTCCAGCTGCGCCAGGGAAGCACAGGCAACCTCGGAGTC \\
\hline A4_S166E_Fw & GAACCGGCACCCCCAGAACCAGAGTCCCATTC \\
\hline A4_S166E_Rv & GAATGGGACTCTGGTTCTGGGGGTGCCGGTTC \\
\hline A4_S166A_Fw & GAACCGGCACCCCCAGCCCCAGAGTCCCATTC \\
\hline A4_S166A_Rv & GAATGGGACTCTGGGGCTGGGGGTGCCGGTTC \\
\hline A4_S169E_Fw & CCCCCAAGCCCAGAGGAACATTCACCCAGGGAC \\
\hline A4_S169E_Rv & GTCCCTGGGTGAATGTTCCTCTGGGCTTGGGGG \\
\hline A4_S169A_Fw & CCCCCAAGCCCAGAGGCCCATTCACCCAGGGAC \\
\hline A4_S169A_Rv & GTCCCTGGGTGAATGGGCCTCTGGGCTTGGGGG \\
\hline A4_(S166E)S169E_Fw & CCCCCAGAACCAGAGGAACATTCACCCAGGGAC \\
\hline A4_(S166E)S169E_Rv & GTCCCTGGGTGAATGTTCCTCTGGTTCTGGGGG \\
\hline A4_(S166A)S169A_Fw & CCCCCAGCCCCAGAGGCCCATTCACCCAGGGAC \\
\hline A4_(S166A)S169A_Rv & GTCCCTGGGTGAATGGGCCTCTGGGGCTGGGGG \\
\hline A4_(S166ES169E)S171E_Fw & GAACCAGAGGAACATGAACCCAGGGACCTGGAC \\
\hline A4_(S166ES169E)S171E_Rv & GTCCAGGTCCCTGGGTTCATGTTCCTCTGGTTC \\
\hline A4_(S166AS169A)S171A_Fw & GCCCCAGAGGCCCATGCACCCAGGGACCTGGAC \\
\hline A4_(S166AS169A)S171A_Rv & GTCCAGGTCCCTGGGTGCATGGGCCTCTGGGGC \\
\hline A4_S183E_Fw & GAGGTTTTCTGTGATGAACTGGAGCAGCTGGAG \\
\hline A4_S183E_Rv & CTCCAGCTGCTCCAGTTCATCACAGAAAACCTC \\
\hline A4_S183A_Fw & GAGGTTTTCTGTGATGCCCTGGAGCAGCTGGAG \\
\hline A4_S183A_Rv & CTCCAGCTGCTCCAGGGCATCACAGAAAACCTC \\
\hline A5_iso2_Fw & AAGGCGATATCATGGCGGACACGAGATCCGTG \\
\hline A5_iso2_Rv & GTTCTCGAGTTATCAGTTCAGTTTTCTTCTCCTTCTTTG \\
\hline A5_dTMD_S495X_Fw & TCTTGGTGGCCCTTCGAGATGTGACCTGGTGTGCTAACGTTTGC \\
\hline A5_dTMD_S495X_Rv & GCAAACGTTAGCACACCAGGTCACATCTCGAAGGGCCACCAAGA \\
\hline A5_S123_124A_Fw & CAAAAAGAGTGGCAGGGCTGCTGATATAACCTCAG \\
\hline A5_S123_124A_Rv & CTGAGGTTATATCAGCAGCCCTGCCACTCTTTTTG \\
\hline A5_S136_T137A_Fw & GTAATGTTCTCACTGCTGCTCCAAACGCCAAAACC \\
\hline A5_S136_T137A_Rv & GGTTTTGGGGTTTGGAGCAGCAGTGAGAACATTAC \\
\hline A5_T252E_Fw & CATGTTGAAGATGTTGAAGGAATTCAGCATTTG \\
\hline A5_T252E_Rv & CAAATGCTGAATTCCTTCAACATCTTCAACATG \\
\hline A5_T252A_Fw & CATGTTGAAGATGTTGCAGGAATTCAGCATTTG \\
\hline A5_T252A_Rv & CAAATGCTGAATTCCTGCAACATCTTCAACATG \\
\hline A5_S259E_Fw & CAGCATTTGACAGAGGATTCAGACAGTGAAG \\
\hline A5_S259E_Rv & CTTCACTGTCTGAATCCTCTGTCAAATGCTG \\
\hline A5_S259A_Fw & TTCAGCATTTGACAGCCGATTCAGACAGTGAAGT \\
\hline A5_S259A_Rv & АCTTCACTGTCTGAATCGGCTGTCAAATGCTGAA \\
\hline A5_S261E_Fw & CATTTGACAAGCGATGAAGACAGTGAAGTTTAC \\
\hline A5_S261E_Rv & GTAAACTTCACTGTCTTCATCGCTTGTCAAATG \\
\hline A5_S261A_Fw & CATTTGACAAGCGATGCAGACAGTGAAGTTTAC \\
\hline
\end{tabular}




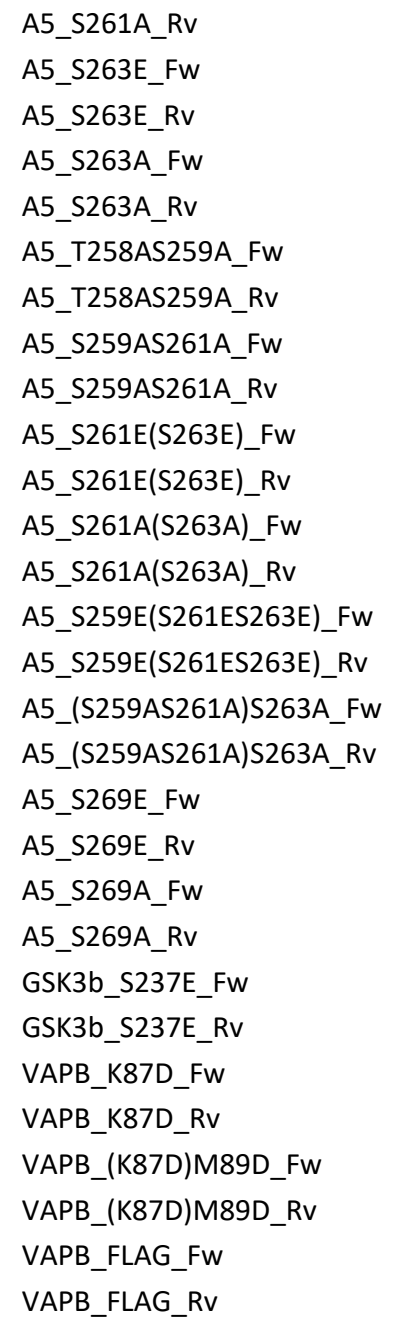

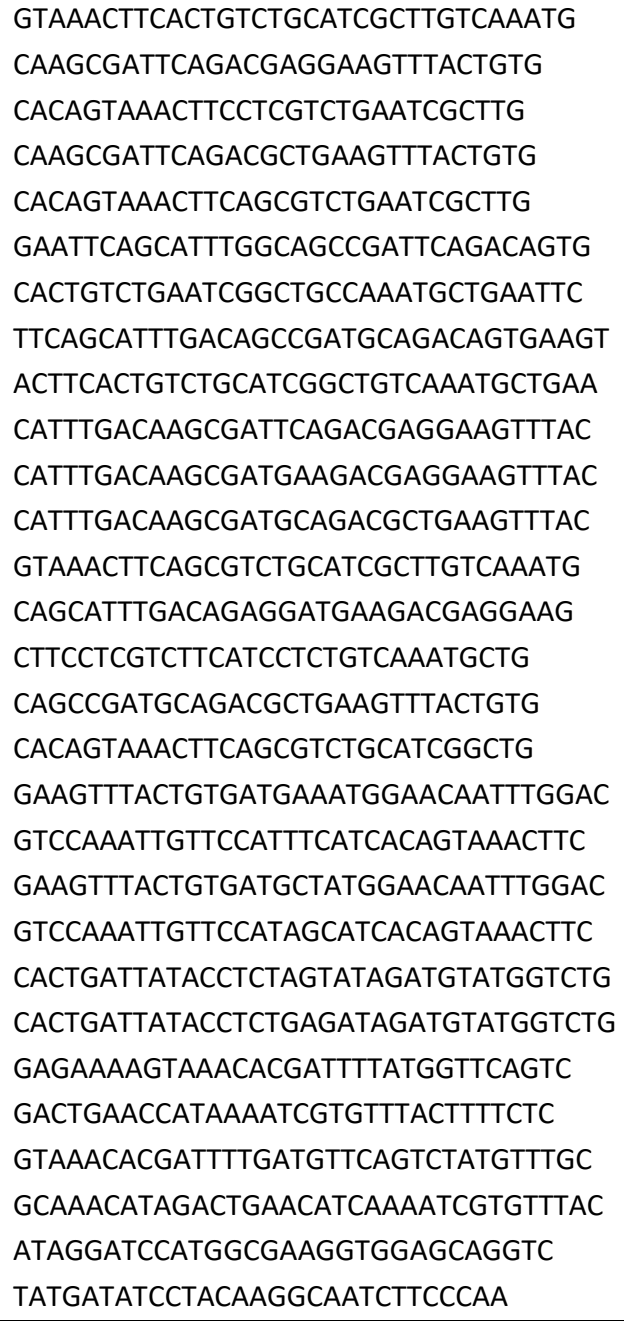

Table S6. Primary and secondary antibodies used in this study.

\begin{tabular}{|c|c|c|c|c|}
\hline \multirow[t]{2}{*}{ Antibody } & \multirow[t]{2}{*}{ Type } & \multicolumn{2}{|l|}{ Dilution } & \multirow[t]{2}{*}{ Source } \\
\hline & & WB & IMF & \\
\hline FLAG & $\mathrm{mc} \mathrm{ms}$ & $1: 2,000$ & $1: 500$ & Sigma F3165 \\
\hline FLAG & pc rb & $1: 1,000$ & & Sigma F7425 \\
\hline Myc & $\mathrm{mc} \mathrm{ms}$ & $1: 1,000$ & $1: 200$ & Santa Cruz sc-40 \\
\hline Myc & $\mathrm{mcrb}$ & $\begin{array}{l}1: 2,000- \\
1: 10,000\end{array}$ & & Abcam ab9106 \\
\hline ACBD5 & pc rb & $1: 500$ & & Cambridge Bioscience HPA012145 \\
\hline ACBD5 control & $\mathrm{pc} \mathrm{rb}$ & $0.5 \mu \mathrm{g} / \mathrm{ml}$ & & Generated for this study by Eurogentec \\
\hline ACBD5 pS269 & pc rb & $0.5 \mu \mathrm{g} / \mathrm{ml}$ & & Generated for this study by Eurogentec \\
\hline$\beta$-catenin & pc rb & $1: 1000$ & & Cell Signalling 9562 \\
\hline$\beta$-catenin pS33ps37 & $\mathrm{mc} \mathrm{ms}$ & $1: 500$ & & Santa Cruz sc-57535 \\
\hline GSK3 $\beta$ & $\mathrm{mc} \mathrm{ms}$ & $1: 500-1,000$ & $1: 50$ & Santa Cruz sc-377213 \\
\hline GSK3 3 pS9 & $\mathrm{mc} \mathrm{ms}$ & $1: 500-1,000$ & & Santa Cruz sc-373800 \\
\hline GSK3 $\beta$ pY216 & $\mathrm{pc} \mathrm{rb}$ & $1: 500-1,000$ & & Abcam ab75745 \\
\hline PEX14 & pc rb & & $1: 14,000$ & $\begin{array}{l}\text { D. Crane, Griffith University, Brisbane, } \\
\text { Australia }\end{array}$ \\
\hline PTPIP51 & pc rb & $1: 500$ & & Sigma HPA009975 \\
\hline VAPB & pc rb & $1: 500$ & & Abcam ab103638 \\
\hline VAPB & $\mathrm{pc} \mathrm{rb}$ & $1: 1,000$ & & Sigma HPA013144 \\
\hline GAPDH & $\mathrm{pc} \mathrm{rb}$ & $1: 10,000$ & & ProSci 3783 \\
\hline
\end{tabular}


bioRxiv preprint doi: https://doi org/10.1101/2021.11.11.467785; this version posted November 12, 2021. The copyright holder for this preprint (which was not certified by peer review) is the author/funder, who has granted bioRxiv a license to display the preprint in perpetuity. It is made available under aCC-BY-NC-ND 4.0 International license.

\begin{tabular}{|c|c|c|c|c|}
\hline atubulin & $\mathrm{mc} \mathrm{ms}$ & $1: 1,000$ & & Sigma T9026 \\
\hline HRP IgG & gt anti-rb & $1: 10,000$ & & Bio-Rad Laboratories 170-6515 \\
\hline HRP IgG & gt anti-ms & $1: 10,000$ & & Bio-Rad Laboratories 170-6516 \\
\hline Alexa Fluor 488 IgG & $\mathrm{dk}$ anti-ms & & $1: 400$ & Molecular Probes A21202 \\
\hline Alexa Fluor 594 lgG & $\mathrm{dk}$ anti-rb & & $1: 1,000$ & Molecular Probes A21207 \\
\hline
\end{tabular}

Abbreviations: dk, donkey; gt, goat; HRP, horseradish peroxidase; IMF, immunofluorescence; mc, monoclonal; $\mathrm{ms}$, mouse; pc, polyclonal; rb, rabbit; WB, Western blot. 


\section{REFERENCES}

Andrés-Benito, P., E. Gelpi, M. Jové, N. Mota-Martorell, È. Obis, M. Portero-Otin, M. Povedano, A. Pujol, R. Pamplona, and I. Ferrer. 2021. Lipid alterations in human frontal cortex in ALS-FTLDTDP43 proteinopathy spectrum are partly related to peroxisome impairment. Neuropathol. Appl. Neurobiol. 47:544-563. doi:10.1111/NAN.12681.

Asare, A., J. Levorse, and E. Fuchs. 2017. Coupling organelle inheritance with mitosis to balance growth and differentiation. Science (80-. ). 355:eaah4701. doi:10.1126/science.aah4701.

Bian, Y., C. Song, K. Cheng, M. Dong, F. Wang, J. Huang, D. Sun, L. Wang, M. Ye, and H. Zou. 2014. An enzyme assisted RP-RPLC approach for in-depth analysis of human liver phosphoproteome. J. Proteomics. 96:253-262. doi:10.1016/j.jprot.2013.11.014.

Bishop, A., M. Kamoshita, J.B. Passmore, C. Hacker, T.A. Schrader, H.R. Waterham, J.L. Costello, and M. Schrader. 2019. Fluorescent tools to analyse peroxisome-ER interactions in mammalian cells. Contact. 2:1-14. doi:10.1177/2515256419848641.

Bonekamp, N.A., K. Vormund, R. Jacob, and M. Schrader. 2010. Dynamin-like protein 1 at the Golgi complex: A novel component of the sorting/targeting machinery en route to the plasma membrane. Exp. Cell Res. 316:3454-3467. doi:10.1016/J.YEXCR.2010.07.020.

Burnett, S.F., J.C. Farré, T.Y. Nazarko, and S. Subramani. 2015. Peroxisomal Pex3 activates selective autophagy of peroxisomes via interaction with the pexophagy receptor Atg30. J. Biol. Chem. 290:8623-8631. doi:10.1074/jbc.M114.619338.

Cabukusta, B., I. Berlin, D.M. van Elsland, I. Forkink, M. Spits, A.W.M. de Jong, J.J.L.L. Akkermans, R.H.M. Wijdeven, G.M.C. Janssen, P.A. van Veelen, and J. Neefjes. 2020. Human VAPome analysis reveals MOSPD1 and MOSPD3 as membrane contact site proteins interacting with FFAT-related FFNT motifs. Cell Rep. 33:108475. doi:10.1016/j.celrep.2020.108475.

Chang, C.-L., A. V Weigel, M.S. Ioannou, H.A. Pasolli, C.S. Xu, D.R. Peale, G. Shtengel, M. Freeman, H.F. Hess, C. Blackstone, and J. Lippincott-Schwartz. 2019. Spastin tethers lipid droplets to peroxisomes and directs fatty acid trafficking through ESCRT-III. J. Cell Biol. 218:2583-2599. doi:10.1083/jcb.201902061.

Chu, B.-B., Y.-C. Liao, W. Qi, C. Xie, X. Du, J. Wang, H. Yang, H.-H. Miao, B.-L. Li, and B.-L. Song. 2015. Cholesterol transport through lysosome-peroxisome membrane contacts. Cell. 161:291-306. doi:10.1016/j.cell.2015.02.019.

Costello, J.L., I.G. Castro, F. Camões, T.A. Schrader, D. McNeall, J. Yang, E.-A. Giannopoulou, S. Gomes, V. Pogenberg, N.A. Bonekamp, D. Ribeiro, M. Wilmanns, G. Jedd, M. Islinger, and M. Schrader. 2017a. Predicting the targeting of tail-anchored proteins to subcellular compartments in mammalian cells. J. Cell Sci. 130:1675-1687. doi:10.1242/jcs.200204.

Costello, J.L., I.G. Castro, C. Hacker, T.A. Schrader, J. Metz, D. Zeuschner, A.S. Azadi, L.F. Godinho, V. Costina, P. Findeisen, A. Manner, M. Islinger, and M. Schrader. 2017b. ACBD5 and VAPB mediate membrane associations between peroxisomes and the ER. J. Cell Biol. 216:331-342. doi:10.1083/jcb.201607055.

Costello, J.L., I.G. Castro, T.A. Schrader, M. Islinger, and M. Schrader. 2017c. Peroxisomal ACBD4 interacts with VAPB and promotes ER-peroxisome associations. Cell Cycle. 16:1039-1045. doi:10.1080/15384101.2017.1314422.

Darwisch, W., M. von Spangenberg, J. Lehmann, Ö. Singin, G. Deubert, S. Kühl, J. Roos, H. Horstmann, C. Körber, S. Hoppe, H. Zheng, T. Kuner, M.L. Pras-Raves, A.H.C. van Kampen, H.R. Waterham, K. V. Schwarz, J.G. Okun, C. Schultz, F.M. Vaz, and M. Islinger. 2020. Cerebellar and hepatic alterations in ACBD5-deficient mice are associated with unexpected, distinct alterations in cellular lipid homeostasis. Commun. Biol. 3:1-19. doi:10.1038/s42003-020-01442-x.

Dong, R., Y. Saheki, S. Swarup, L. Lucast, J.W. Harper, and P. De Camilli. 2016. Endosome-ER Contacts Control Actin Nucleation and Retromer Function through VAP-Dependent Regulation of PI4P. Cell. 166:408-423. doi:10.1016/j.cell.2016.06.037.

Ferdinandusse, S., K.D. Falkenberg, J. Koster, P.A. Mooyer, R. Jones, C.W.T. van Roermund, A. Pizzino, M. Schrader, R.J.A. Wanders, A. Vanderver, and H.R. Waterham. 2017. ACBD5 
deficiency causes a defect in peroxisomal very long-chain fatty acid metabolism. J. Med. Genet. 54:330-337. doi:10.1136/jmedgenet-2016-104132.

Frame, S., and P. Cohen. 2001. GSK3 takes centre stage more than 20 years after its discovery. Biochem. J. 359:1-16. doi:10.1042/0264-6021:3590001.

Furuita, K., J.G. Jee, H. Fukada, M. Mishima, and C. Kojima. 2010. Electrostatic interaction between oxysterol-binding protein and VAMP-associated protein a revealed by NMR and mutagenesis studies. J. Biol. Chem. 285:12961-12970. doi:10.1074/jbc.M109.082602.

Goto, A., X. Liu, C.-A. Robinson, and N.D. Ridgway. 2012. Multisite phosphorylation of oxysterolbinding protein regulates sterol binding and activation of sphingomyelin synthesis. Mol. Biol. Cell. 23:3624-3635. doi:10.1091/mbc.E12-04-0283.

Graves, H.K., S. Jangam, K.L. Tan, A. Pignata, E.S. Seto, S. Yamamoto, and M.F. Wangler. 2020. A genetic screen for genes that impact peroxisomes in Drosophila identifies candidate genes for human disease. G3 Genes/Genomes/Genetics. 10:69-77. doi:10.1534/g3.119.400803.

Guillén-Samander, A., M. Leonzino, M.G. Hanna, N. Tang, H. Shen, and P. De Camilli. 2021. VPS13D bridges the ER to mitochondria and peroxisomes via Miro. J. Cell Biol. 220:e202010004. doi:10.1083/jcb.202010004.

Herzog, K., M.L. Pras-Raves, S. Ferdinandusse, M.A.T. Vervaart, A.C.M. Luyf, A.H.C. van Kampen, R.J.A. Wanders, H.R. Waterham, and F.M. Vaz. 2018. Functional characterisation of peroxisomal $\beta$-oxidation disorders in fibroblasts using lipidomics. J. Inherit. Metab. Dis. 41:479-487. doi:10.1007/s10545-017-0076-9.

Hornbeck, P. V., B. Zhang, B. Murray, J.M. Kornhauser, V. Latham, and E. Skrzypek. 2015. PhosphoSitePlus, 2014: Mutations, PTMs and recalibrations. Nucleic Acids Res. 43:D512-D520. doi:10.1093/nar/gku1267.

Hua, R., D. Cheng, É. Coyaud, S. Freeman, E. Di Pietro, Y. Wang, A. Vissa, C.M. Yip, G.D. Fairn, N. Braverman, J.H. Brumell, W.S. Trimble, B. Raught, and P.K. Kim. 2017. VAPs and ACBD5 tether peroxisomes to the ER for peroxisome maintenance and lipid homeostasis. J. Cell Biol. 216:367-377. doi:10.1083/jcb.201608128.

Humphrey, S.J., O. Karayel, D.E. James, and M. Mann. 2018. High-throughput and high-sensitivity phosphoproteomics with the EasyPhos platform. Nat. Protoc. 13:1897-1916. doi:10.1038/s41596-018-0014-9.

Johnson, B., A.N. Leek, L. Solé, E.E. Maverick, T.P. Levine, and M.M. Tamkun. 2018. Kv2 potassium channels form endoplasmic reticulum/plasma membrane junctions via interaction with VAPA and VAPB. Proc. Natl. Acad. Sci. 115:E7331-E7340. doi:10.1073/pnas.1805757115.

Joshi, S., G. Agrawal, and S. Subramani. 2012. Phosphorylation-dependent Pex11p and Fis1p interaction regulates peroxisome division. Mol. Biol. Cell. 23:1307-1315. doi:10.1091/mbc.E1109-0782.

Kaiser, S.E., J.H. Brickner, A.R. Reilein, T.D. Fenn, P. Walter, and A.T. Brunger. 2005. Structural basis of FFAT motif-mediated ER targeting. Structure. 13:1035-1045. doi:10.1016/j.str.2005.04.010.

Kinoshita, E., E. Kinoshita-Kikuta, K. Takiyama, and T. Koike. 2006. Phosphate-binding tag, a new tool to visualize phosphorylated proteins. Mol. Cell. Proteomics. 5:749-757. doi:10.1074/mcp.T500024-MCP200.

Kirmiz, M., N.C. Vierra, S. Palacio, and J.S. Trimmer. 2018. Identification of VAPA and VAPB as Kv2 channel-interacting proteins defining endoplasmic reticulum-plasma membrane junctions in mammalian brain neurons. J. Neurosci. 38:7562-7584. doi:10.1523/JNEUROSCI.0893-18.2018.

Knoblach, B., and R.A. Rachubinski. 2010. Phosphorylation-dependent activation of peroxisome proliferator protein PEX11 controls peroxisome abundance. J. Biol. Chem. 285:6670-6680. doi:10.1074/jbc.M109.094805.

Koch, J., R.G. Feichtinger, P. Freisinger, M. Pies, F. Schrödl, A. luso, W. Sperl, J.A. Mayr, H. Prokisch, and T.B. Haack. 2016. Disturbed mitochondrial and peroxisomal dynamics due to loss of MFF causes Leigh-like encephalopathy, optic atrophy and peripheral neuropathy. J. Med. Genet. 53:270-278. doi:10.1136/JMEDGENET-2015-103500. 
Kumagai, K., M. Kawano-Kawada, and K. Hanada. 2014. Phosphoregulation of the ceramide transport protein CERT at serine 315 in the interaction with VAMP-associated protein (VAP) for inter-organelle trafficking of ceramide in mammalian cells. J. Biol. Chem. 289:10748-10760. doi:10.1074/jbc.M113.528380.

Loewen, C.J.R., A. Roy, and T.P. Levine. 2003. A conserved ER targeting motif in three families of lipid binding proteins and in Opi1p binds VAP. EMBO J. 22:2025-2035. doi:10.1093/emboj/cdg201.

Longoni, P., D. Douchi, F. Cariti, G. Fucile, and M. Goldschmidt-Clermont. 2015. Phosphorylation of the Lhcb2 isoform of Light Harvesting Complex II is central to state transitions. Plant Physiol. 169:2874-2883. doi:10.1104/pp.15.01498.

Madeira, F., Y.M. Park, J. Lee, N. Buso, T. Gur, N. Madhusoodanan, P. Basutkar, A.R.N. Tivey, S.C. Potter, R.D. Finn, and R. Lopez. 2019. The EMBL-EBI search and sequence analysis tools APIs in 2019. Nucleic Acids Res. 47:W636-W641. doi:10.1093/nar/gkz268.

Di Mattia, T., A. Martinet, S. Ikhlef, A.G. McEwen, Y. Nominé, C. Wendling, P. Poussin-Courmontagne, L. Voilquin, P. Eberling, F. Ruffenach, J. Cavarelli, J. Slee, T.P. Levine, G. Drin, C. Tomasetto, and F. Alpy. 2020. FFAT motif phosphorylation controls formation and lipid transfer function of inter-organelle contacts. EMBO J. 39:e104369. doi:10.15252/embj.2019104369.

Mikitova, V., and T.P. Levine. 2012. Analysis of the key elements of FFAT-like motifs identifies new proteins that potentially bind VAP on the ER, including two AKAPs and FAPP2. PLoS One. 7:e30455. doi:10.1371/journal.pone.0030455.

Murphy, S.E., and T.P. Levine. 2016. VAP, a versatile access point for the endoplasmic reticulum: Review and analysis of FFAT-like motifs in the VAPome. Biochim. Biophys. Acta-Mol. Cell Biol. Lipids. 1861:952-961. doi:10.1016/j.bbalip.2016.02.009.

Nthiga, T.M., B. Kumar Shrestha, E. Sjøttem, J. Bruun, K. Bowitz Larsen, Z. Bhujabal, T. Lamark, and T. Johansen. 2020. CALCOCO 1 acts with VAMP -associated proteins to mediate ER -phagy. EMBO J. 39:e103649. doi:10.15252/embj.2019103649.

Oeljeklaus, S., A. Schummer, T. Mastalski, H.W. Platta, and B. Warscheid. 2016. Regulation of peroxisome dynamics by phosphorylation. Biochim. Biophys. Acta - Mol. Cell Res. 1863:10271037. doi:10.1016/j.bbamcr.2015.12.022.

Okumoto, K., M. El Shermely, M. Natsui, H. Kosako, R. Natsuyama, T. Marutani, and Y. Fujiki. 2020. The peroxisome counteracts oxidative 1 stresses by suppressing catalase import via pex14 phosphorylation. Elife. 9:e55896. doi:10.7554/ELIFE.55896.

Perez-Riverol, Y., A. Csordas, J. Bai, M. Bernal-Llinares, S. Hewapathirana, D.J. Kundu, A. Inuganti, J. Griss, G. Mayer, M. Eisenacher, E. Pérez, J. Uszkoreit, J. Pfeuffer, T. Sachsenberg, S. Yilmaz, S. Tiwary, J. Cox, E. Audain, M. Walzer, A.F. Jarnuczak, T. Ternent, A. Brazma, and J.A. Vizcaíno. 2019. The PRIDE database and related tools and resources in 2019: Improving support for quantification data. Nucleic Acids Res. 47:D442-D450. doi:10.1093/nar/gky1106.

Prinz, W.A., A. Toulmay, and T. Balla. 2020. The functional universe of membrane contact sites. Nat. Rev. Mol. Cell Biol. 21:7-24. doi:10.1038/s41580-019-0180-9.

Ranea-Robles, P., N. Launay, M. Ruiz, N.Y. Calingasan, M. Dumont, A. Naudí, M. Portero-Otín, R. Pamplona, I. Ferrer, M.F. Beal, S. Fourcade, and A. Pujol. 2018. Aberrant regulation of the GSK33/NRF2 axis unveils a novel therapy for adrenoleukodystrophy. EMBO Mol. Med. 10:e8604. doi:10.15252/EMMM.201708604.

Rappsilber, J., Y. Ishihama, and M. Mann. 2003. Stop And Go Extraction tips for matrix-assisted laser desorption/ionization, nanoelectrospray, and LC/MS sample pretreatment in proteomics. Anal. Chem. 75:663-670. doi:10.1021/ac026117i.

Sawyer, E.M., P.R. Joshi, V. Jorgensen, J. Yunus, L.E. Berchowitz, and E. Ünal. 2019. Developmental regulation of an organelle tether coordinates mitochondrial remodeling in meiosis. J. Cell Biol. 218:559-579. doi:10.1083/jcb.201807097.

Schrader, M., J.L. Costello, L.F. Godinho, A.S. Azadi, and M. Islinger. 2016. Proliferation and fission of peroxisomes - An update. Biochim. Biophys. Acta. 1863:971-983. doi:10.1016/j.bbamcr.2015.09.024. 
Schrader, M., M. Kamoshita, and M. Islinger. 2020. Organelle interplay - Peroxisome interactions in health and disease. J. Inherit. Metab. Dis. 43:71-89. doi:10.1002/jimd.12083.

Schummer, A., R. Maier, S. Gabay-Maskit, T. Hansen, W.W.D. Mühlhäuser, I. Suppanz, A. Fadel, M. Schuldiner, W. Girzalsky, S. Oeljeklaus, E. Zalckvar, R. Erdmann, and B. Warscheid. 2020. Pex14p phosphorylation modulates import of citrate synthase 2 into peroxisomes in Saccharomyces cerevisiae. Front. Cell Dev. Biol. 8:549451. doi:10.3389/fcell.2020.549451.

Shai, N., E. Yifrach, C.W.T. van Roermund, N. Cohen, C. Bibi, L. IJlst, L. Cavellini, J. Meurisse, R. Schuster, L. Zada, M.C. Mari, F.M. Reggiori, A.L. Hughes, M. Escobar-Henriques, M.M. Cohen, H.R. Waterham, R.J.A. Wanders, M. Schuldiner, and E. Zalckvar. 2018. Systematic mapping of contact sites reveals tethers and a function for the peroxisome-mitochondria contact. Nat. Commun. 9:1761. doi:10.1038/s41467-018-03957-8.

Shamseldin, H.E., M. Alshammari, T. Al-Sheddi, M.A. Salih, H. Alkhalidi, A. Kentab, G.M. Repetto, M. Hashem, and F.S. Alkuraya. 2012. Genomic analysis of mitochondrial diseases in a consanguineous population reveals novel candidate disease genes. J. Med. Genet. 49:234-241. doi:10.1136/JMEDGENET-2012-100836.

Sharma, K., R.C.J. D'Souza, S. Tyanova, C. Schaab, J.R. Wiśniewski, J. Cox, and M. Mann. 2014. Ultradeep human phosphoproteome reveals a distinct regulatory nature of Tyr and Ser/Thrbased signaling. Cell Rep. 8:1583-1594. doi:10.1016/j.celrep.2014.07.036.

Silva, B.S.C., L. DiGiovanni, R. Kumar, R.E. Carmichael, P.K. Kim, and M. Schrader. 2020. Maintaining social contacts: The physiological relevance of organelle interactions. Biochim. Biophys. Acta Mol. Cell Res. 1867:118800. doi:10.1016/j.bbamcr.2020.118800.

da Silva, T.F., J. Eira, A.T. Lopes, A.R. Malheiro, V. Sousa, A. Luoma, R.L. Avila, R.J.A. Wanders, W.W. Just, D.A. Kirschner, M.M. Sousa, and P. Brites. 2014. Peripheral nervous system plasmalogens regulate Schwann cell differentiation and myelination. J. Clin. Invest. 124:2560-2570. doi:10.1172/JCI72063.

Slee, J.A., and T.P. Levine. 2019. Systematic prediction of FFAT motifs across eukaryote proteomes identifies nucleolar and eisosome proteins with the predicted capacity to form bridges to the endoplasmic reticulum. Contact. 2:1-21. doi:10.1177/2515256419883136.

Stanhope, R., E. Flora, C. Bayne, and I. Derré. 2017. IncV, a FFAT motif-containing Chlamydia protein, tethers the endoplasmic reticulum to the pathogen-containing vacuole. Proc. Natl. Acad. Sci. 114:12039-12044. doi:10.1073/pnas.1709060114.

Stoica, R., S. Paillusson, P. Gomez-Suaga, J.C. Mitchell, D.H. Lau, E.H. Gray, R.M. Sancho, G. VizcayBarrena, K.J. De Vos, C.E. Shaw, D.P. Hanger, W. Noble, and C.C. Miller. 2016. ALS/FTDassociated FUS activates GSK-3 $\beta$ to disrupt the VAPB-PTPIP51 interaction and ER-mitochondria associations. EMBO Rep. 17:1326-1342. doi:10.15252/embr.201541726.

Stoica, R., K.J. De Vos, S. Paillusson, S. Mueller, R.M. Sancho, K.-F. Lau, G. Vizcay-Barrena, W.-L. Lin, Y.-F. Xu, J. Lewis, D.W. Dickson, L. Petrucelli, J.C. Mitchell, C.E. Shaw, and C.C.J. Miller. 2014. ERmitochondria associations are regulated by the VAPB-PTPIP51 interaction and are disrupted by ALS/FTD-associated TDP-43. Nat. Commun. 5:3996. doi:10.1038/ncomms4996.

Thomas, A.S., A.M. Krikken, I.J. Van Der Klei, and C.P. Williams. 2015. Phosphorylation of Pex11p does not regulate peroxisomal fission in the yeast Hansenula polymorpha. Sci. Rep. 5:11493. doi:10.1038/srep11493.

Ullah, S., S. Lin, Y. Xu, W. Deng, L. Ma, Y. Zhang, Z. Liu, and Y. Xue. 2016. DbPAF: An integrative database of protein phosphorylation in animals and fungi. Sci. Rep. 6:23534. doi:10.1038/srep23534.

Valm, A.M., S. Cohen, W.R. Legant, J. Melunis, U. Hershberg, E. Wait, A.R. Cohen, M.W. Davidson, E. Betzig, and J. Lippincott-Schwartz. 2017. Applying systems-level spectral imaging and analysis to reveal the organelle interactome. Nature. 546:162-167. doi:10.1038/nature22369.

Wanders, R.J.A., and H.R. Waterham. 2006. Biochemistry of mammalian peroxisomes revisited. Annu. Rev. Biochem. 75:295-332. doi:10.1146/annurev.biochem.74.082803.133329.

Wanders, R.J.A., H.R. Waterham, and S. Ferdinandusse. 2016. Metabolic interplay between 
peroxisomes and other subcellular organelles including mitochondria and the endoplasmic reticulum. Front. Cell Dev. Biol. 3:83. doi:10.3389/fcell.2015.00083.

Wang, B., R. Malik, E.A. Nigg, and R. Körner. 2008. Evaluation of the Low-Specificity Protease Elastase for Large-Scale Phosphoproteome Analysis. Anal. Chem. 80:9526-9533. doi:10.1021/AC801708P.

Weber-Boyvat, M., H. Kentala, J. Lilja, T. Vihervaara, R. Hanninen, Y. Zhou, J. Peränen, T.A. Nyman, J. Ivaska, and V.M. Olkkonen. 2015. OSBP-related protein 3 (ORP3) coupling with VAMPassociated protein A regulates R-Ras activity. Exp. Cell Res. 331:278-291. doi:10.1016/j.yexcr.2014.10.019.

Wiechmann, S., B. Ruprecht, T. Siekmann, R. Zheng, M. Frejno, E. Kunold, T. Bajaj, D.P. Zolg, S.A. Sieber, N.C. Gassen, and B. Kuster. 2021. Chemical Phosphoproteomics Sheds New Light on the Targets and Modes of Action of AKT Inhibitors. ACS Chem. Biol. 16:631-641. doi:10.1021/ACSCHEMBIO.0C00872.

Xiao, J., J. Luo, A. Hu, T. Xiao, M. Li, Z. Kong, L. Jiang, Z. Zhou, Y. Liao, C. Xie, B. Chu, H. Miao, B. Li, X. Shi, and B.-L. Song. 2019. Cholesterol transport through the peroxisome-ER membrane contacts tethered by PI(4,5)P2 and extended synaptotagmins. Sci. China. Life Sci. 62:11171135. doi:10.1007/s11427-019-9569-9.

Yamashita, K., S. Tamura, M. Honsho, H. Yada, Y. Yagita, H. Kosako, and Y. Fujiki. 2020. Mitotic phosphorylation of Pex14p regulates peroxisomal import machinery. J. Cell Biol. 219:e202001003. doi:10.1083/JCB.202001003.

Zhang, J., D.N. Tripathi, J. Jing, A. Alexander, J. Kim, R.T. Powell, R. Dere, J. Tait-Mulder, J.H. Lee, T.T. Paull, R.K. Pandita, V.K. Charaka, T.K. Pandita, M.B. Kastan, and C.L. Walker. 2015. ATM functions at the peroxisome to induce pexophagy in response to ROS. Nat. Cell Biol. 17:12591269. doi:10.1038/ncb3230. 


\section{FIGURE LEGENDS}

Figure 1. The interaction between ACBD5 and VAPB is sensitive to phosphatase treatment. (A) Immunoblots of lysates of FLAG-ACBD4 or FLAG-ACBD5 protein expressed in COS-7 cells treated with or without $\lambda$-phosphatase ( $\lambda P P$ ), using conventional and Phos-tag SDS-PAGE. $\alpha$ Tubulin and Myc (unspecific band) served as loading control. (B) Binding assay with FLAG-ACBD4 or FLAG-ACBD5 expressed in COS-7 cells and recombinant GST-VAPB. FLAG-ACBD4/5 was treated with $\lambda$ PP. Constructs with mutations in the FFAT-like motif (mFFAT) were used as a negative control. Samples were immunoprecipitated (IP) (GST-TRAP) and immunoblotted using FLAG/VAPB antibodies. (C) MycACBD5 was expressed in COS-7 cells, and lysates treated with or without $\lambda$ PP. Myc-ACBD5 was immunoprecipitated and endogenous bound VAPB detected by immunoblotting using Myc/VAPB antibodies. Data were analyzed by a two-tailed unpaired t-test; ${ }^{*}, \mathrm{P}<0.05$. Error bars represent SEM, with five independent experiments. Total VAPB (IP fraction) was normalized against Myc-ACBD5 (IP fraction). (D) ACBD5 protein sequence. Phosphorylation sites identified by database search (Hornbeck et al., 2015; Ullah et al., 2016) and our own mass spectrometry (MS)-based analyses are indicated (DOI: 10.6019/PXD018005) (filled boxes). Coloured boxes enclosing sequence regions indicate different protein domains (colours as in Fig. 2A). Bold regions represent peptides identified by MS ($\mathrm{TiO}_{2}$ and $+\mathrm{TiO}_{2}$ ). The FFAT-like motif is underlined. (E) The FFAT-like motif region of ACBD4 was replaced with that of ACBD5 (ACBD5 FFAT). FLAG-ACBD4/5 constructs were expressed in COS-7 cells and immunoprecipitated to detect endogenous bound VAPB using FLAG/VAPB antibodies. WT, wild type.

Figure 2. Phospho-mutants of the acidic tract alter the ACBD5-VAPB interaction and its phosphatasesensitivity. (A) Schematic overview of ACBD5 domain structure, including the amino acid sequences of the FFAT-like motif region, with the phosphorylation sites mutated in this study in bold. (B) Schematic model of the interaction between the ACBD5 FFAT-like motif and the VAPB MSP domain. The interaction occurs in two steps (Furuita et al., 2010): (1) An initial non-specific electrostatic interaction between the acidic tract of the FFAT motif and the basic electropositive surface of the MSP domain; (2) A specific binding of the FFAT core region to the FFAT-binding site of the MSP domain, which consists of an electropositive face. (C, D, E) ACBD5 constructs with non-phosphorylatable (S $\rightarrow$ A) and phosphomimetic $(S \rightarrow E$ ) residues in the acidic tract were generated and expressed in COS-7 cells. The proteins were immunoprecipitated and endogenous bound VAPB detected by immunoblotting using Myc/VAPB antibodies. (C) Data were analyzed by one-way analysis of variance with Dunnett's multiple comparison test. Total VAPB (IP fraction) was normalized against total VAPB (input) and Myc-ACBD5 (IP fraction). (D, E) Lysates were treated with or without $\lambda$ PP before immunoprecipitation. Data were analyzed by one-way analysis of variance with Dunnett's multiple comparison test (D) or a two-tailed unpaired t-test (E). Total VAPB (IP fraction) was normalized against Myc-ACBD5 (IP fraction). Normalized VAPB signal in the treated sample was then calculated as a percentage of normalized VAPB signal in the untreated sample. ns, not significant; *, $\mathrm{P}<0.05$; ${ }^{* *}$, $\mathrm{P}$ $<0.001 ; * * *, \mathrm{P}<0.0001$. Results of at least three independent IPs were quantified. Error bars represent SEM. WT, wild type.

Figure 3. The serine in the FFAT core of ACBD5 is phosphorylated and inhibits VAPB binding. (A) ACBD5 constructs with non-phosphorylatable $(S \rightarrow A)$ and phosphomimetic $(S \rightarrow E)$ residues upstream (T252) or within the FFAT core (S269) were generated and expressed in COS-7 cells. The proteins were 
immunoprecipitated and endogenous bound VAPB detected by immunoblotting using Myc/VAPB antibodies. Data were analyzed by one-way analysis of variance with Dunnett's multiple comparison test; ns, not significant. Error bars represent SEM. Results of three independent IPs were quantified. Total VAPB (IP fraction) was normalized against total VAPB (input) and Myc-ACBD5 (IP fraction). (B) The serine residue within the core of the ACBD5 FFAT-like motif (position 5, S269) binds VAPB in a hydrophobic pocket (Kaiser et al., 2005; Furuita et al., 2010). Phosphorylation at this position likely causes steric hindrance, inhibiting the FFAT-VAPB interaction. (C) Lysates of COS-7 cells expressing Myc-ACBD5 were treated with or without $\lambda$ PP before immunoprecipitation. Phosphorylation of ACBD5 at S269 was examined by immunoblotting using ACBD5 pS269/ACBD5 control/Myc antibodies. (D) Myc-ACBD5 WT, S269A phospho-mutant or control vector (Myc) was expressed in COS-7 cells. The proteins were immunoprecipitated and phosphorylation of ACBD5 at S269 was examined by immunoblotting using ACBD5 pS269/ACBD5 control/Myc antibodies. The ACBD5 antibodies were generated against a peptide of the same region. (E) Myc-VAPB and FLAG-ACBD5 were immunoprecipitated from COS-7 cells separately and subsequently incubated together to allow MycVAPB and FLAG-ACBD5 to interact. ACBD5 S269 phosphorylation in the VAPB-bound (IP) and nonVAPB-bound fraction was examined by immunoblotting using ACBD5 pS269/FLAG/Myc antibodies. WT, wild type; mFFAT, mutated FFAT motif (negative control).

Figure 4. ACBD5 phospho-sites alter peroxisome-ER associations. (A) Representative electron micrographs of peroxisome-ER contacts in ACBD5 KO HeLa cells transfected with Myc-ACBD5 WT, S259A, S261A, S259A/S261A/S263A, S269E or control vector (Myc). (B) Quantitative analysis of the mean population of peroxisomes associated with the ER. (C) Assessment of the mean peroxisome membrane surface in direct contact with the ER. Data were analyzed by one-way analysis of variance with Dunnett's multiple comparison test; ns, not significant; *, $\mathrm{P}<0.05 ;{ }^{* *}, \mathrm{P}<0.01 ;{ }^{* *}, \mathrm{P}<0.001$. Error bars represent SEM. Results of four grids per condition. (D) Immunoblots of cell lysates from ACBD5 KO HeLa cells expressing the indicated Myc-ACBD5 constructs. $\alpha$ Tubulin and Myc (unspecific band) served as loading control. Bars: $200 \mathrm{~nm}$. WT, wild type.

Figure 5. GSK3 $\beta$ affects the ACBD5-VAPB interaction. (A) Myc-VAPB (or Myc control vector) was expressed in the absence or presence of GSK3 $\beta$ in COS-7 cells. Myc-VAPB was immunoprecipitated and endogenous bound ACBD5 and PTPIP51 detected by immunoblotting using Myc/ACBD5/PTPIP51 antibodies. Results of three independent IPs were quantified. ACBD5/PTPIP51 (IP fraction) was normalized against total ACBD5/PTPIP51 (input) and Myc-VAPB (IP fraction). (B) FLAG-ACBD5 was expressed in HEK293T cells. Cells were treated with $10 \mu \mathrm{M}$ CHIR (GSK3 $\beta$ inhibitor) or DMSO (control) for $16 \mathrm{~h}$. FLAG-ACBD5 was immunoprecipitated and endogenous bound VAPB detected by immunoblotting using FLAG/VAPB antibodies. Inhibition of GSK3 $\beta$ by CHIR was confirmed using GSK3 $\beta / G S K 3 \beta$ pY216/ $\beta$-catenin antibodies (arrow indicates GSK3 $\beta$ ). VAPB (IP fraction) was normalized against total VAPB (input) and FLAG-ACBD5 (IP fraction). $n=5-8$ of three independent IPs. Data were analyzed by a two-tailed unpaired t-test; ${ }^{* *}, \mathrm{P}<0.01 ;{ }^{* *}, \mathrm{P}<0.001$. Error bars represent SEM.

Figure 6. GSK3 $\beta$ associates with ACBD5 and VAPB. (A) GSK3 $\beta$ (S237E 'mFFAT') was co-expressed with FLAG-VAPB, FLAG-ACBD5 WT/mFFAT/ATMD, or control vector (FLAG) in COS-7 cells. FLAGVAPB/ACBD5 were immunoprecipitated and bound GSK3 $\beta$ and endogenous VAPB detected by immunoblotting using FLAG/GSK3 $\beta$ /VAPB antibodies. Asterisk indicates unspecific band (due to reprobing of the blot). (B) GSK3 $\beta$ was co-expressed with FLAG-VAPB (K87D/M89D mMSP), or control 
vector (FLAG) in COS-7 cells. FLAG-VAPB w immunoprecipitated and bound GSK3 $\beta$ and endogenous ACBD5 detected by immunoblotting using FLAG/GSK3B/ACBD5 antibodies. (C) FLAG-ACBD5 constructs with non-phosphorylatable $(S \rightarrow A)$ residues in the acidic tract were co-expressed with GSK3 $\beta$ in COS7 cells. FLAG-ACBD5 was immunoprecipitated and phosphorylation at S269 (pS269) was detected by immunoblotting using ACBD5 pS269 and ACBD5 control antibodies (generated against a peptide of the same region). Bound GSK3 $\beta$ was detected by immunoblotting using ACBD5 control/GSK3 $\beta$ antibodies. GSK3 $\beta$ (IP fraction) was normalized against total GSK3 $\beta$ (input) and ACBD5 control (IP fraction). Data were analyzed by one-way analysis of variance with Dunnett's multiple comparison test; ns, not significant; ${ }^{* * *}, \mathrm{P}<0.001 ;{ }^{* * *}, \mathrm{P}<0.0001$. Error bars represent SEM. Results of three independent IPs were quantified. WT, wild type; mFFAT, mutated FFAT motif; TMD, transmembrane domain.

Figure 7. GSK3 $\beta$ modulates the ACBD5-VAPB interaction via S269. (A) GSK3 $\beta$ (K85A) was coexpressed with Myc-ACBD5 WT or S269A in COS-7 cells. Myc-ACBD5 was immunoprecipitated and endogenous bound VAPB detected by immunoblotting using Myc/VAPB antibodies. VAPB (IP fraction) was normalized against total VAPB (input) and Myc-ACBD5 (IP fraction). Phosphorylation of ACBD5 S269 (pS269) was detected by immunoblotting using ACBD5 pS269/Myc antibodies. GSK3 $\beta$ catalytic (in)activity was confirmed using GSK3 $\beta / G S K 3 \beta$ pY216/ $\beta$-catenin antibodies. Data were analyzed by one-way analysis of variance with Tukey's multiple comparisons test. Results of four independent IPs were quantified. (B) Recombinant His-ACBD5 was incubated in the absence or presence of recombinant GST-GSK3 $\beta$. Phosphorylation of ACBD5 at S269 was examined by immunoblotting using ACBD5 pS269/ACBD5 control antibodies. (C) Representative electron micrographs of peroxisome-ER contacts in COS-7 cells transfected with a catalytically inactive GSK3 $\beta$ (GSK3 $\beta$ K85A) or GSK3 $\beta$ WT. (D) Assessment of the mean peroxisome membrane surface in direct contact with the ER. Quantitative analysis of the mean population of peroxisomes associated with the ER. Data were analyzed by a twotailed unpaired t-test. Results of four grids per condition. ns, not significant; *, $\mathrm{P}<0.05 ;{ }^{* * *}, \mathrm{P}<0.001$; $* * * *, \mathrm{P}<0.0001$. Error bars represent SEM. Bars: $200 \mathrm{~nm}$. WT, wild type.

Figure 8. Model of ACBD5-VAPB interaction and regulation at the peroxisome-ER interface. Peroxisomal ACBD5 and ER-resident VAPB interact via the FFAT and MSP domains to enable peroxisome-ER contacts. The FFAT-MSP interaction involves the FFAT core (VYCDSME) and flanking acidic tract. ACBD5 phosphorylation (P) can promote (green; acidic tract) or inhibit (red; FFAT core) VAPB interaction. GSK3 $\beta$ regulates the ACBD5-VAPB interaction via phosphorylation of the serine in the FFAT core. ACB, acyl-CoA binding domain.

Supplementary Figure S1. The ACBD5-VAPB binding, but not the ACBD4-VAPB interaction, is sensitive to phosphatase treatment. (A) FLAG-ACBD4/5 was expressed in COS-7 cells, and lysates were treated with or without $\lambda$ PP. FLAG-ACBD4/5 was immunoprecipitated and endogenous bound VAPB detected by immunoblotting using FLAG/VAPB antibodies. Constructs with mutations in the FFAT-like motif (mFFAT) were used as a negative control. (B) Myc-ACBD5 phospho-mutants were expressed in COS-7 cells and immunoprecipitated to detect endogenous bound VAPB using Myc/VAPB antibodies. WT, wild type.

Supplementary Figure S2. Potential phosphorylation sites involved in the ACBD4/5-VAPB interaction. (A) Schematic overview of replacement of the ACBD4 FFAT-like motif region (bold) by that 
of ACBD5. (B) Schematic overview of ACBD4 and ACBD5 domain structure, including the amino acid sequences of the FFAT-like motif region, with the phosphorylation sites mutated in this study in bold. (C) Alignment of the FFAT-like motif region of ACBD4 and ACBD5 from Hs, Homo sapiens (human); Rn, Rattus norvegicus (rat); Mm, Mus musculus (mouse); $\mathrm{Cl}$, Canis lupus familiaris (dog); Fp, Falco peregrinus (Falcon); Xt, Xenopus tropicalis (frog); Dr, Danio rerio (zebrafish). Conservation of the phosphorylation sites reported in this study is indicated in bold. (D) Overview of the FFAT-like motif region of ACBD4 and ACBD5 and the constructs used in this study. Mutated residues are indicated in bold.

Supplementary Figure S3. ACBD4 phosphomimetic mutants increase VAPB interaction. (A) Subcellular localisation of ACBD4/5 constructs. COS-7 cells transfected with Myc-ACBD5 WT, mFFAT, S269E, S259A/S261A/S263A or $\triangle T$ TMD; or FLAG-ACBD4 WT, S183E or S166A/S169A/S171A, were immunolabelled with PEX14 (peroxisomal marker) and Myc/FLAG antibodies. Bars: $10 \mu \mathrm{m}$ (main), 2.5 $\mu \mathrm{m}$ (insets). (B, C) ACBD4 constructs with non-phosphorylatable $(S \rightarrow A)$ and phosphomimetic $(S \rightarrow E)$ residues upstream (S166/S169/S171) or within the FFAT core (S183) were generated and expressed in COS-7 cells. The FLAG-tagged proteins were immunoprecipitated and endogenous bound VAPB detected by immunoblotting using FLAG/VAPB antibodies. (B) Data were analyzed by one-way analysis of variance with Dunnett's multiple comparison test. Total VAPB (IP fraction) was normalized against total VAPB (input) and FLAG-ACBD4 (IP fraction). Results of five independent IPs were quantified. (D) Quantification of peroxisome morphology in Myc-ACBD5 (S269E) or FLAG-ACBD4 (S183E) transfected COS-7 cells. Data were analyzed by one-way analysis of variance with Tukey's multiple comparisons test. $\mathrm{n}=400$ per condition, from four replicates. $\mathrm{ns}$, not significant; ${ }^{*}, \mathrm{P}<0.05 ;{ }^{* * * *}, \mathrm{P}<0.0001$. Error bars represent SEM. WT, wild type; mFFAT, mutated FFAT motif; TMD, transmembrane domain.

Supplementary Figure S4. GSK3 $\beta$ expression alters peroxisome morphology in MFF-deficient fibroblasts. (A) GSK3 $\beta$ expression increased phosphorylation of its substrate $\beta$-catenin, resulting in its degradation in COS-7 cells, as assessed by immunoblotting using GSK3 $\beta / G S K 3 \beta$ pY216/ $\beta$-catenin/ $\beta$ catenin pS33pS37 antibodies. GAPDH served as loading control. GSK3 $\beta$ K85A, catalytically inactive mutant; WT, wild type. (B) Myc-VAPB WT or mMSP (K87D/M89D), a mutant that cannot bind FFAT motifs, was co-expressed with FLAG-ACBD5 (or control vector (FLAG)). Myc-VAPB was immunoprecipitated and bound FLAG-ACBD5 detected by immunoblotting using Myc/FLAG antibodies. (C) Peroxisome morphology in MFF-deficient fibroblasts expressing GSK3 $\beta$. Fixed cells were immunolabelled with PEX14 (peroxisomal marker) and GSK3 $\beta$ antibodies. Data were analyzed by a two-tailed unpaired t-test; $* * * *, \mathrm{P}<0.0001$. Error bars represent SEM. $\mathrm{n}=800$ per condition, from two independent experiments. Bars: $10 \mu \mathrm{m}$.

\section{Supplementary Figure S5. Examples of proteins with a serine/threonine residue at position $\mathbf{5}$ of the} FFAT core. A screen of human proteins with predicted FFAT motifs (score $\leq 2.5$ ) identified by (Slee and Levine, 2019) revealed additional proteins, as well as ACBD4 and ACBD5, with a serine/threonine residue at position 5 of the FFAT core. These residues and (predicted) FFAT motifs showed conservation between species. Some of the serine/threonine residues at position 5 have been shown to be phosphorylated (indicated by an asterisk) (Hornbeck et al., 2015). The FFAT scores of the shown sequences are indicated (Murphy and Levine, 2016). FFAT motifs with a score of $\leq 3.5$ are highlighted. Light green, acidic tract of the FFAT motif; dark green, FFAT core; orange, serine/threonine residue at position 5 of the FFAT core. Hs, Homo sapiens (human); Rn, Rattus norvegicus (rat); Mm, Mus musculus 
bioRxiv preprint doi: https://doi org/10.1101/2021.11.11.467785; this version posted November 12, 2021. The copyright holder for this preprint (which was not certified by peer review) is the author/funder, who has granted bioRxiv a license to display the preprint in perpetuity. It is made available under aCC-BY-NC-ND 4.0 International license.

(mouse); $\mathrm{Cl}$, Canis lupus familiaris (dog); Fp, Falco peregrinus (falcon); $\mathrm{Xt}$, Xenopus tropicalis (frog); $\mathrm{Dr}$, Danio rerio (zebrafish). 
Fiaure 1 bioRxiv preprint doi: https://doi.org/10.1101/2021.11.11.467785; this version posted November 12,2021 . The copyright holder for this preprint (which was not certified by peer review) is the author/funder, who has granted bioRxiv a license to display the preprint in perpetuity. It is made available under aCC-BY-NC-ND 4.0 International license.

A

FLAG-ACBD4

Conventional Phos-tag

Phosphatase inhibitor +-++

$\lambda$-phosphatase

$\alpha-F L A G$
(ACBD4
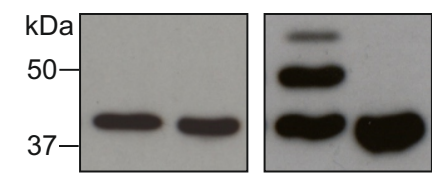

$50-$

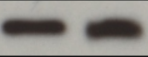

FLAG-ACBD5

Conventional Phos-tag

Phosphatase inhibitor +-++ $\lambda$-phosphatase

$\alpha-F L A G$
(ACBD5)

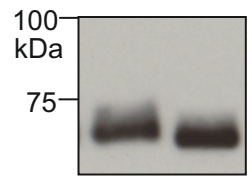

a-Myc

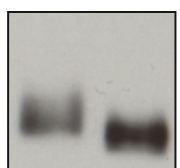

$100-\longrightarrow$
B

FLAG-\#

ACBD4

\begin{tabular}{|c|c|c|c|}
\hline WT & mFFAT & WT & nFFAT \\
\hline & & & \\
\hline . & & & \\
\hline
\end{tabular}

Phosphatase in
$\lambda$-phosphatase
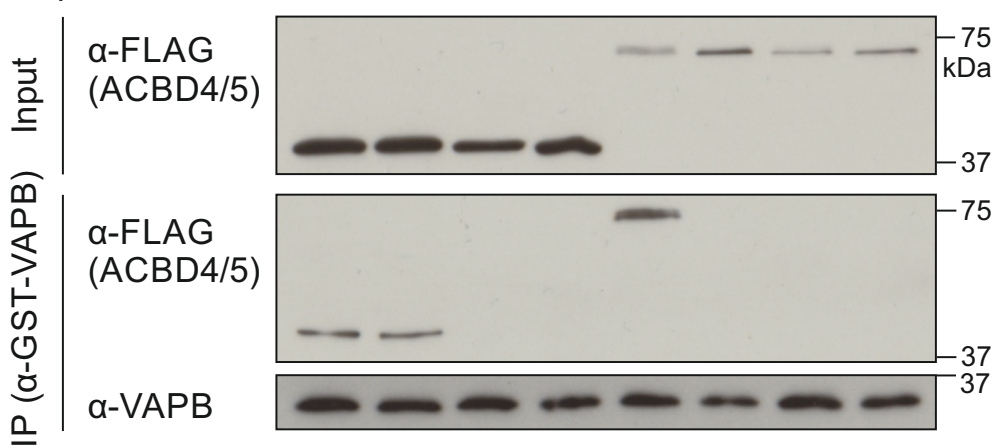

C

Phosphatase inhibitor $\frac{\text { WT }}{+-} \frac{\text { mFFAT }}{+-}$ $\lambda$-phosphatase

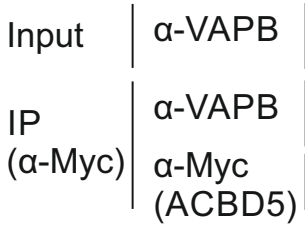

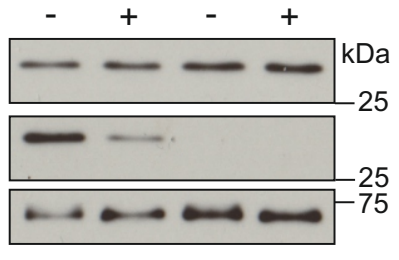

$\lambda$-phosphatase

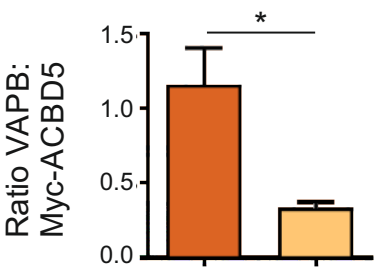

$+$

D 1 MADTRSVHETRFEAVKVIQSLPKNGSFQPTNEMMLKFYSFYKQATEGPCKLSRPGFWDP 60

61 IGRYKWDAWSSLGDMTKEEAMIAYVEEMKKI IETMPMTEKVEELLRVIGPFYEIVEDKKS 120

121 GRSSDITSDLGNVITSTPNAKTVNGKAESSDSGAESEEEEAQEEVKGAEQSDNDKKMMKK 180

181 SADHKNLEVIVTNGYDKDGFVQDIQNDIHASSSINGRSTEEVKPIDENLGQTGKSAVCIH 240

241 QDINDDHVEDVTGIQHLTS SSDEVYCDSMEQFGQEESLDSFTSNNGPFQYYLGGHSSQP 300

301 MENSGFREDIQVPPGNGNIGNMQVVAVEGKGEVKHGGEDGRNNSGAPHEKRGGETDEFS 360

361 NVRRGRGHRMQHLSEGTKGRQVGSGGDGERWGSDRGSRGSLNEQIALVLMRLQEDMQNVL 420

421 QRLQKLETLTALQAKS STSLQTAPQPTSQRPSWWPFEMSPGVLTFAIWPFIAQWLVYL 480

481 Y Y $Q R R R R K L N$

$E$

FLAG-\#

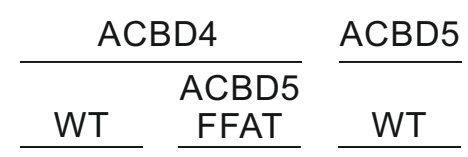

Database

MassSpec

\begin{tabular}{llll} 
& \multicolumn{2}{c}{ ACBD5 } & \\
Phosphatase inhibitor & FFAT & WT \\
& +- & + & +
\end{tabular}

Both

$\lambda$-phosphatase

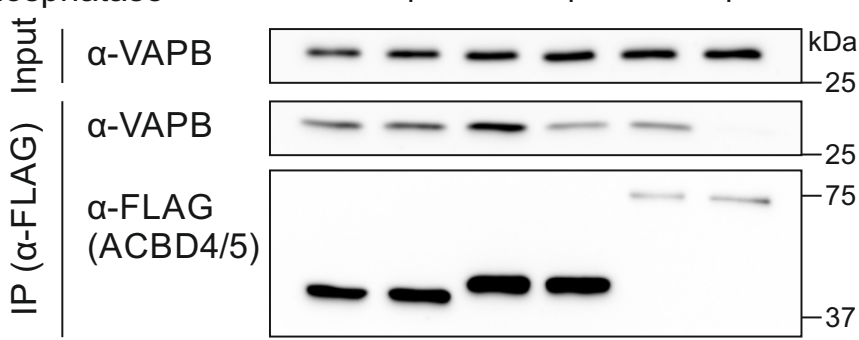


Figure 2 bioRxiv preprint doi: https://doi.org/10.1101/2021.11.11.467785; this version posted November 12, 2021. The copyright holder for this preprint (which was not certified by peer review) is the author/funder, who has granted bioRxiv a license to display the preprint in perpetuity. It is made available under aCC-BY-NC-ND 4.0 International license.

A ACBD5

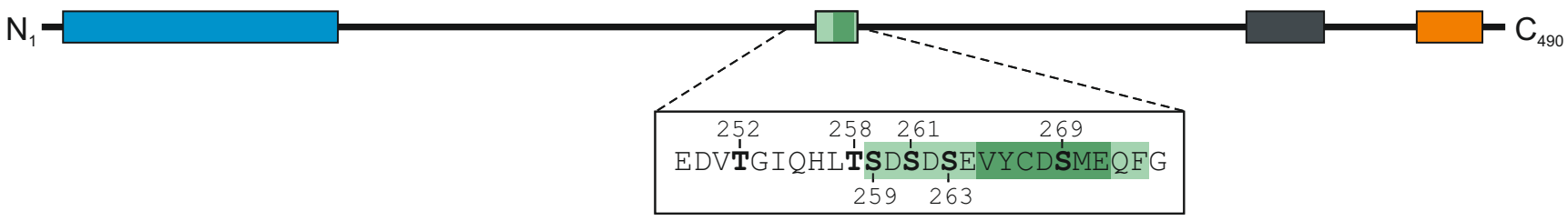

B

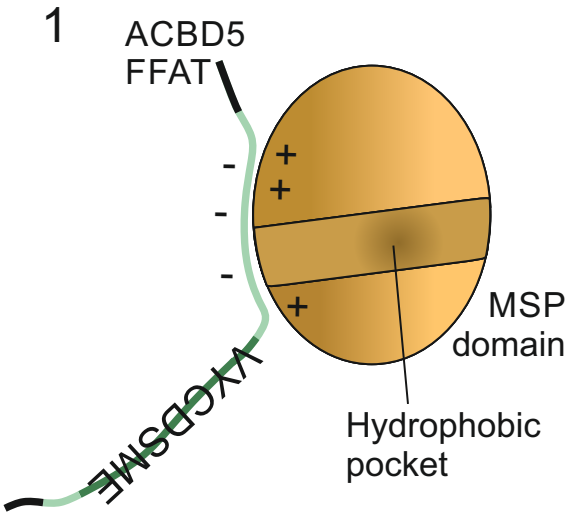

2

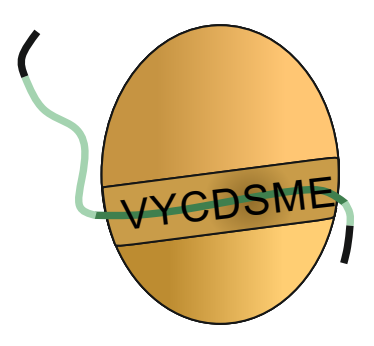

ACB domain

Acidic tract FFAT-like motif Core FFAT-like motif

Coiled-coil region

Transmembrane domain
C

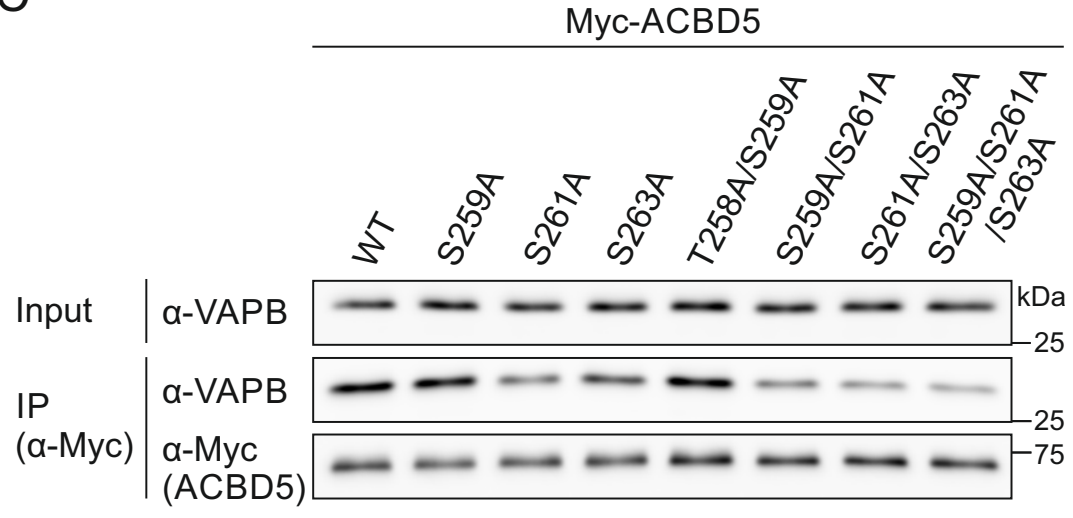

$\mathrm{D}$

Myc-ACBD5
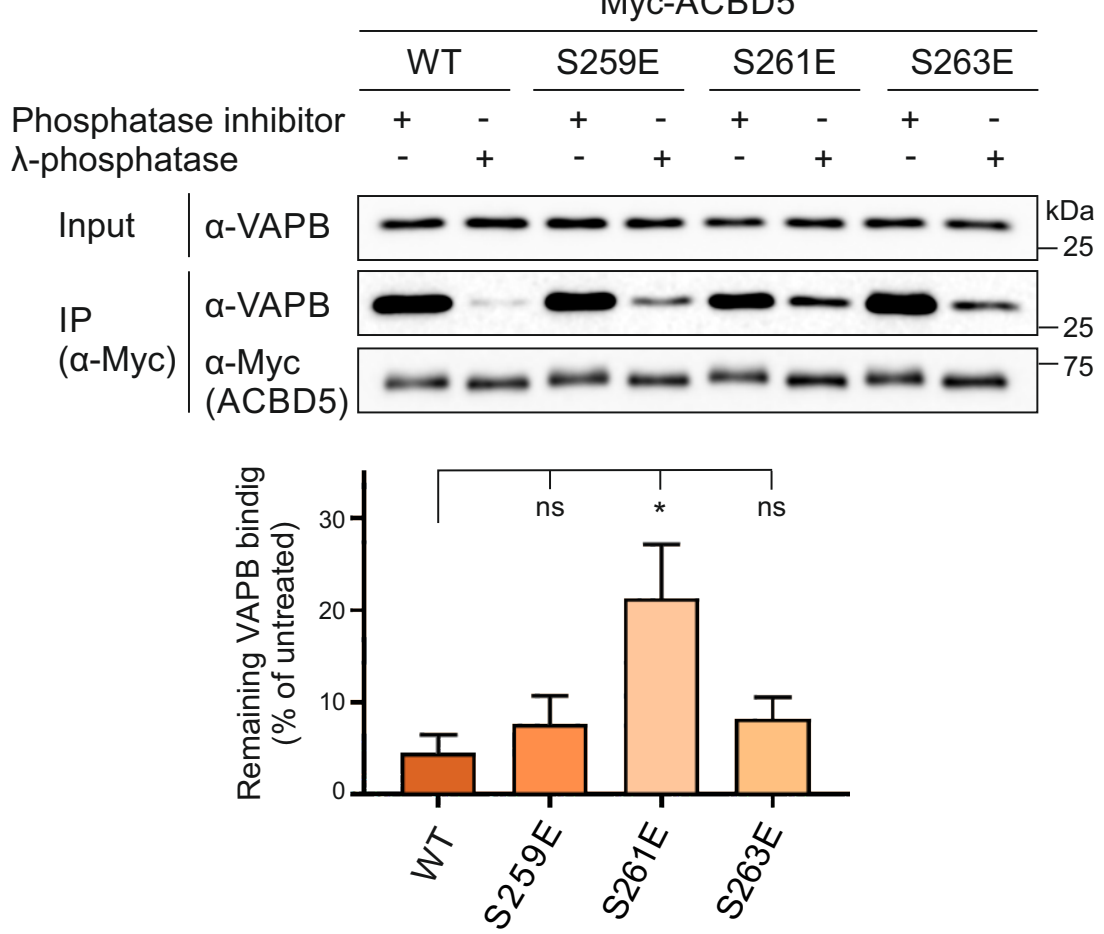

E
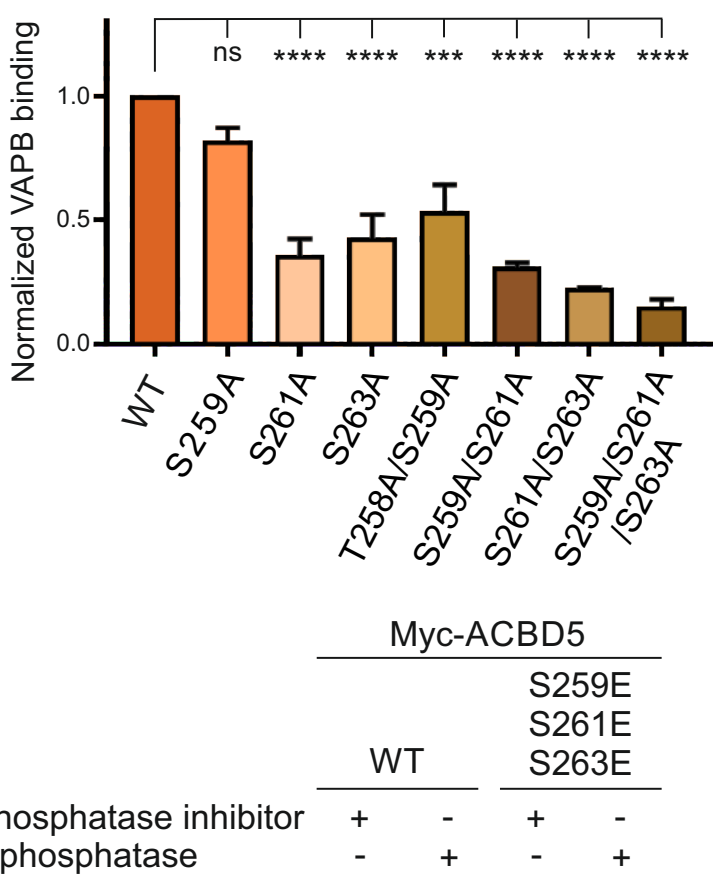

$\lambda$-phosphatase
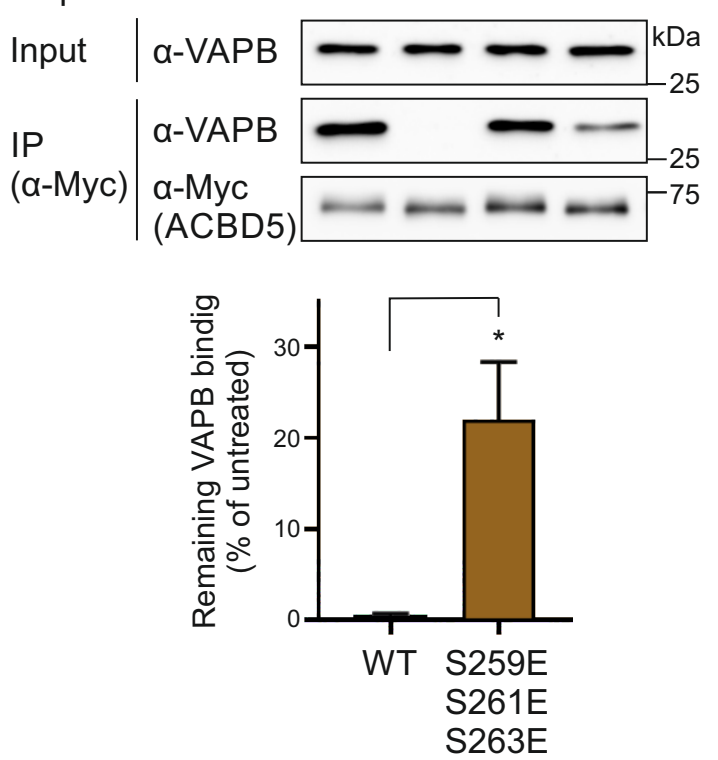
Figure 3 bioRxiv preprint doi: https://doi.org/10.1101/2021.11.11.467785; this version posted November 12, 2021. The copyright holder for this preprint (which was not certified by peer review) is the author/funder, who has granted bioRxiv a license to display the preprint in perpetuity. It is made available under aCC-BY-NC-ND 4.0 International license.

A

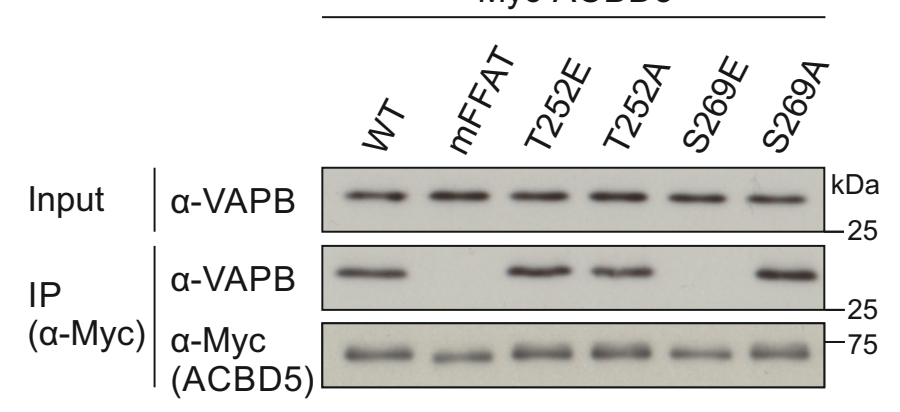

C

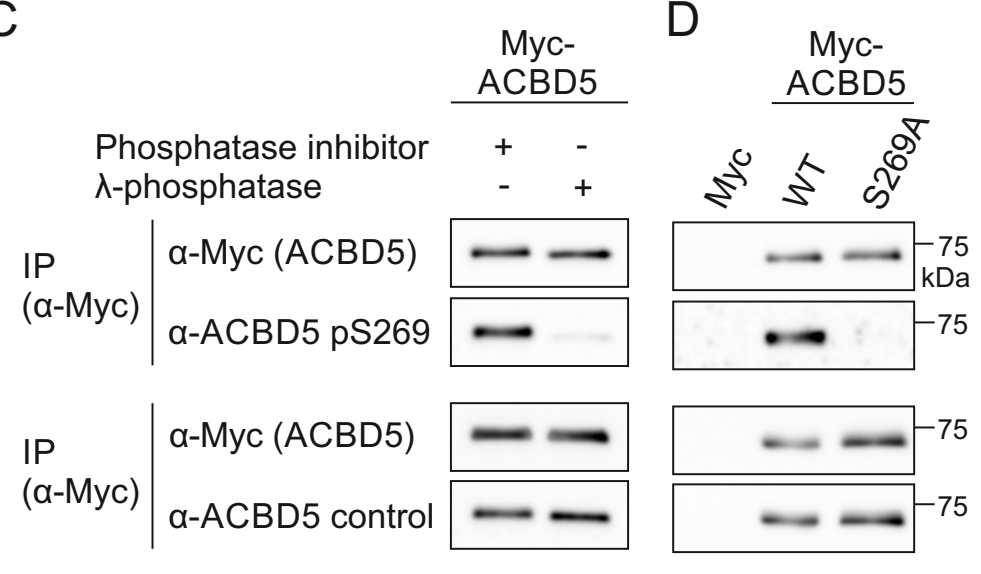

Myc-ACBD5

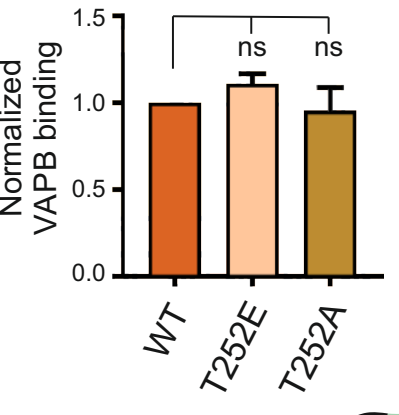

$\mathrm{E}$

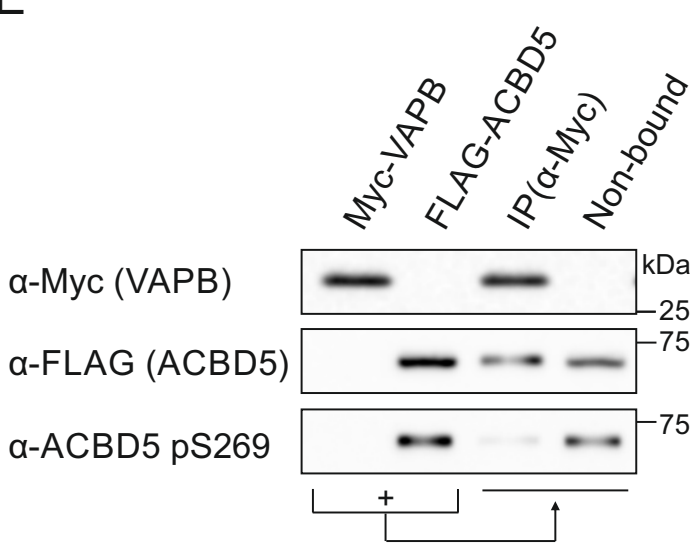

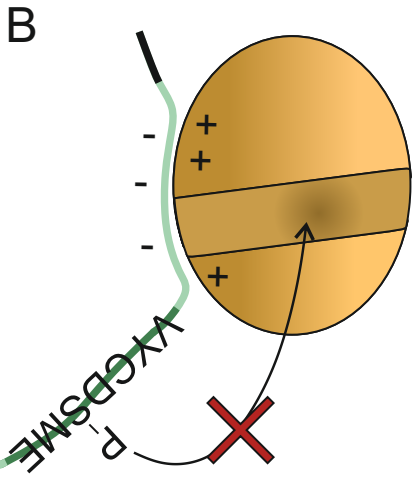


Figure 4 bioRxiv preprint doi: https://doi.org/10.1101/2021.11.11.467785; this version posted November 12, 2021. The copyright holder for this preprint (which was not certified by peer review) is the author/funder, who has granted bioRxiv a license to display the preprint in perpetuity. It is made available under aCC-BY-NC-ND 4.0 International license.

A
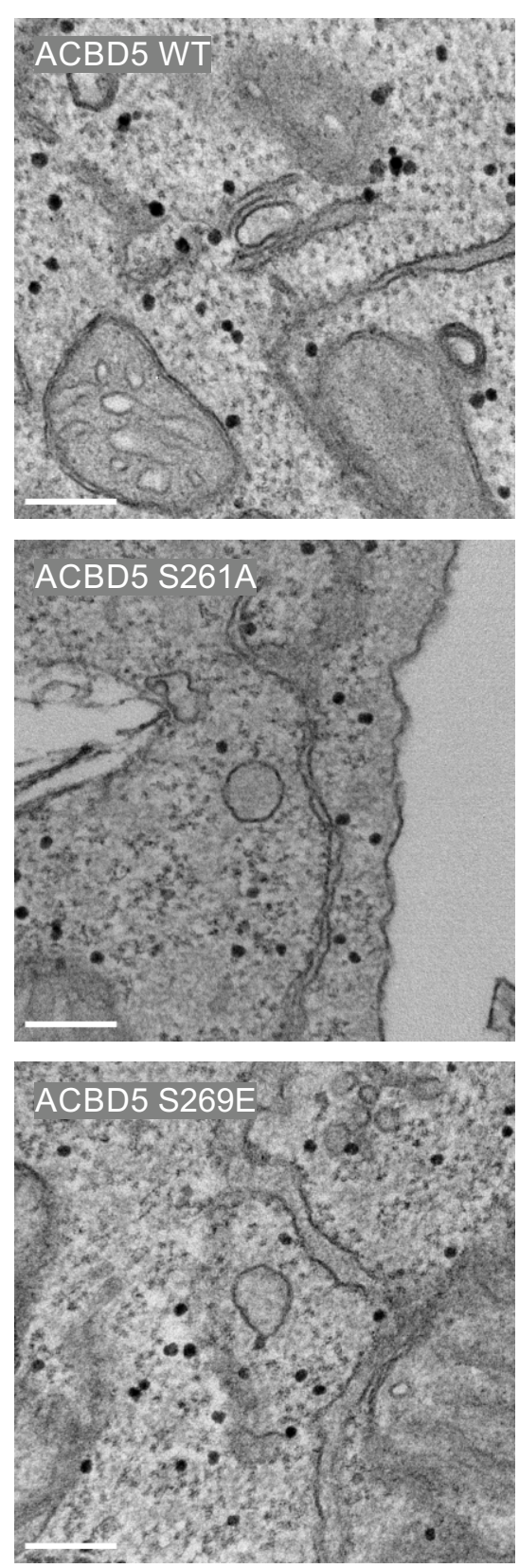
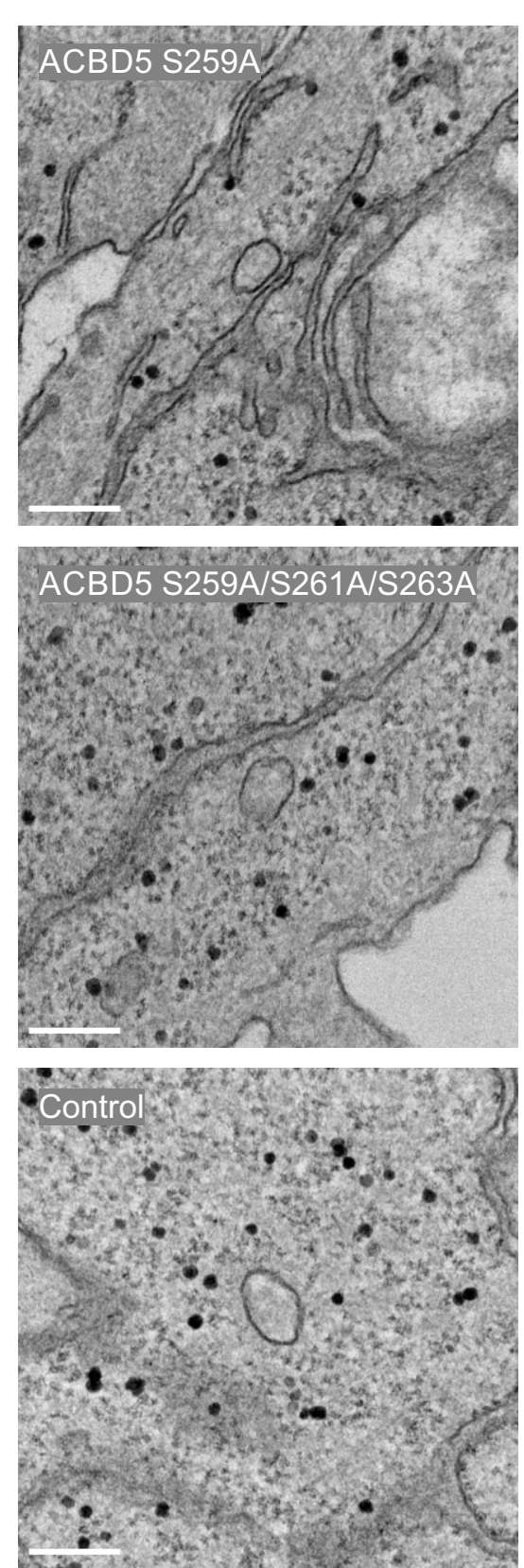

B

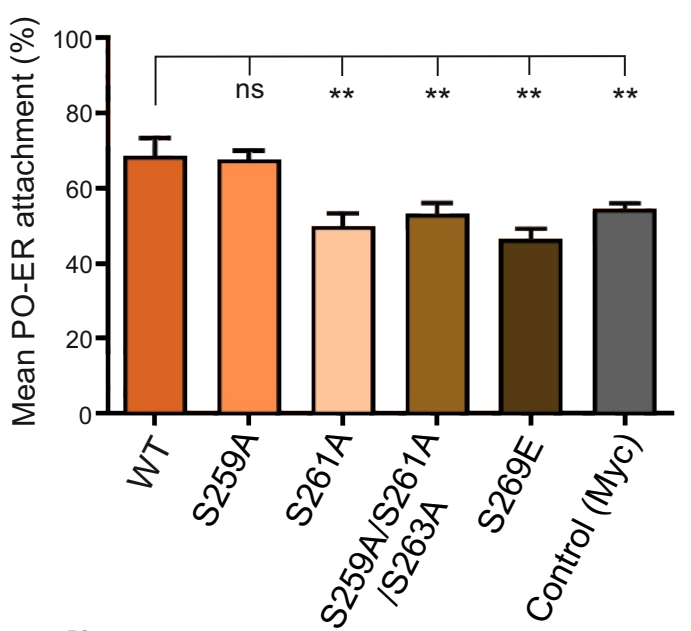

C

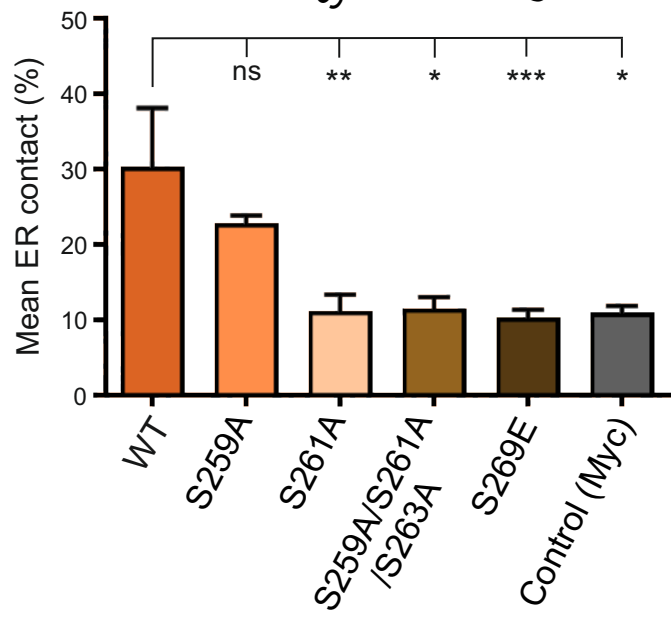

$\mathrm{D}$

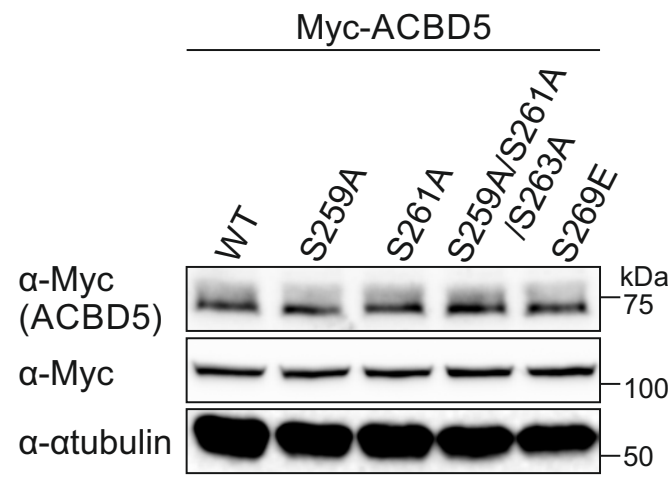


Figure 5 bioRxiv preprint doi: https://doi.org/10.1101/2021.11.11.467785; this version posted November 12, 2021. The copyright holder for this preprint (which was not certified by peer review) is the author/funder, who has granted bioRxiv a license to display the preprint in perpetuity. It is made available under aCC-BY-NC-ND 4.0 International license.

A

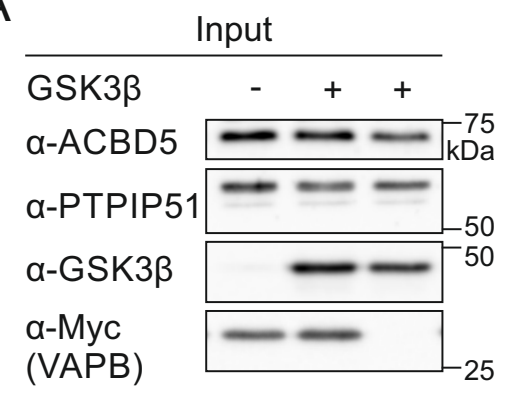

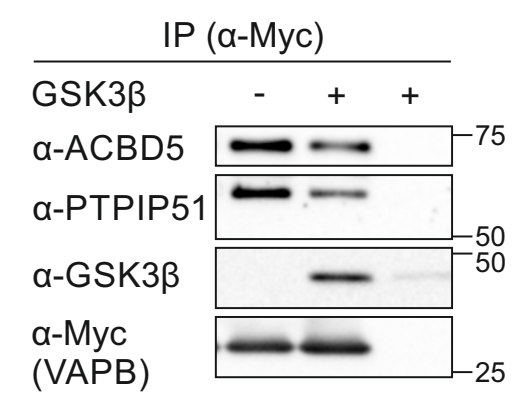

B

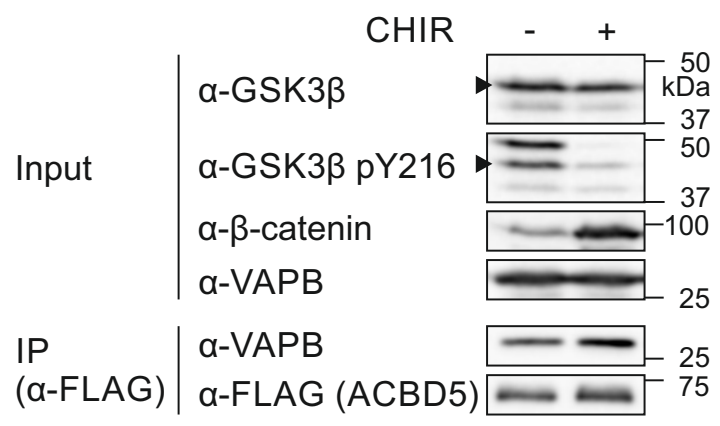

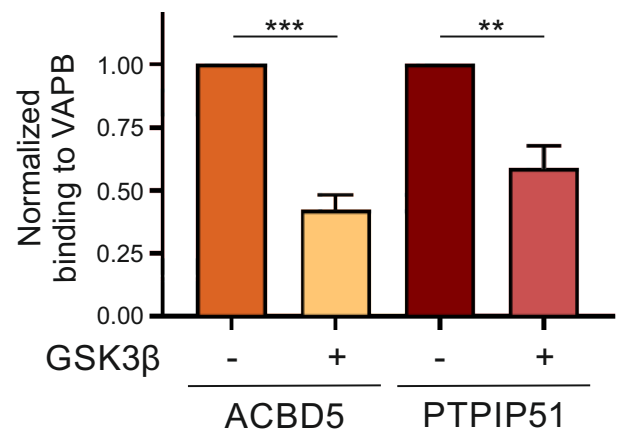

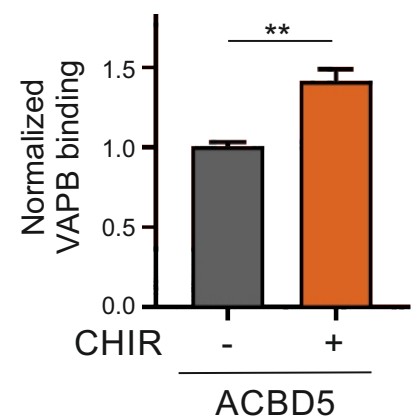


Fiaure 6 bioRxiv preprint doi: https://doi.org/10.1101/2021.11.11.467785; this version posted November 12, 2021. The copyright holder for this preprint (which was not certified by peer review) is the author/funder, who has granted bioRxiv a license to display the preprint in perpetuity. It is made available under aCC-BY-NC-ND 4.0 International license.

A
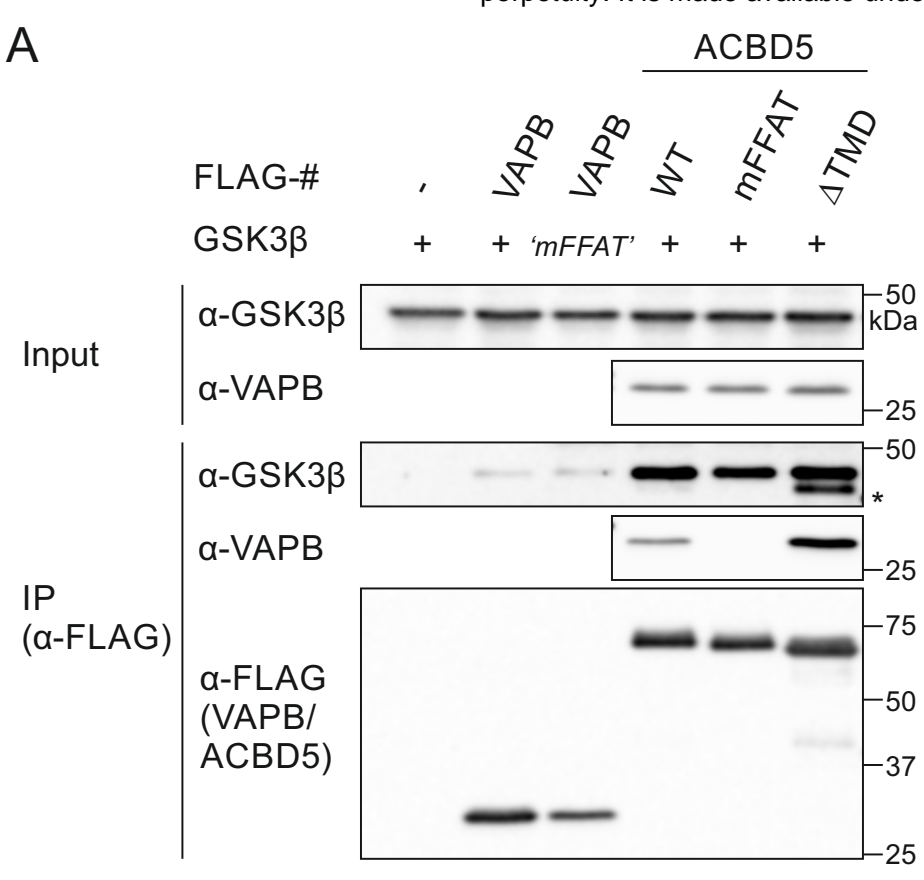

B

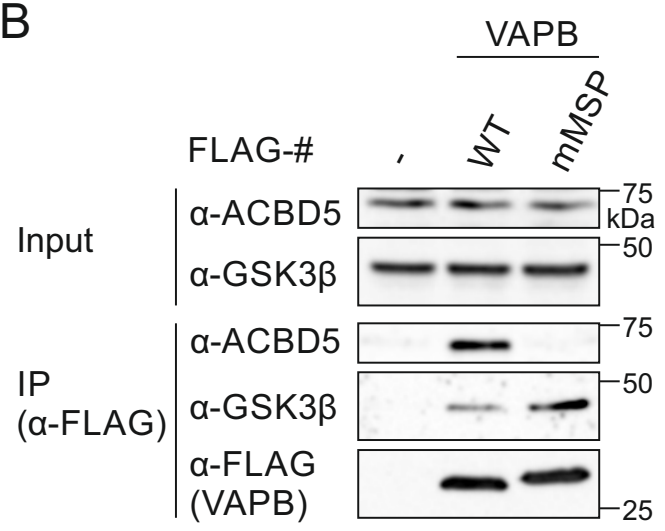

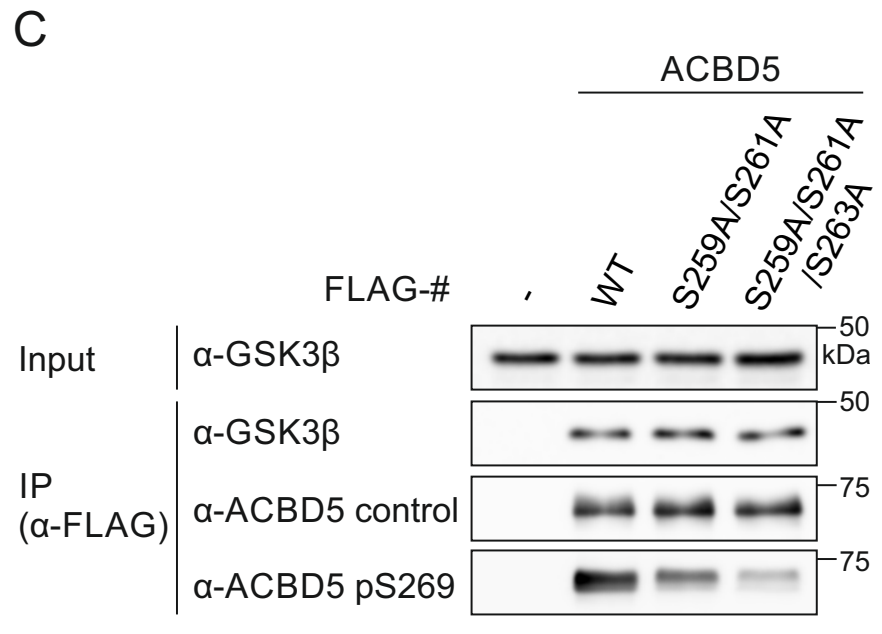
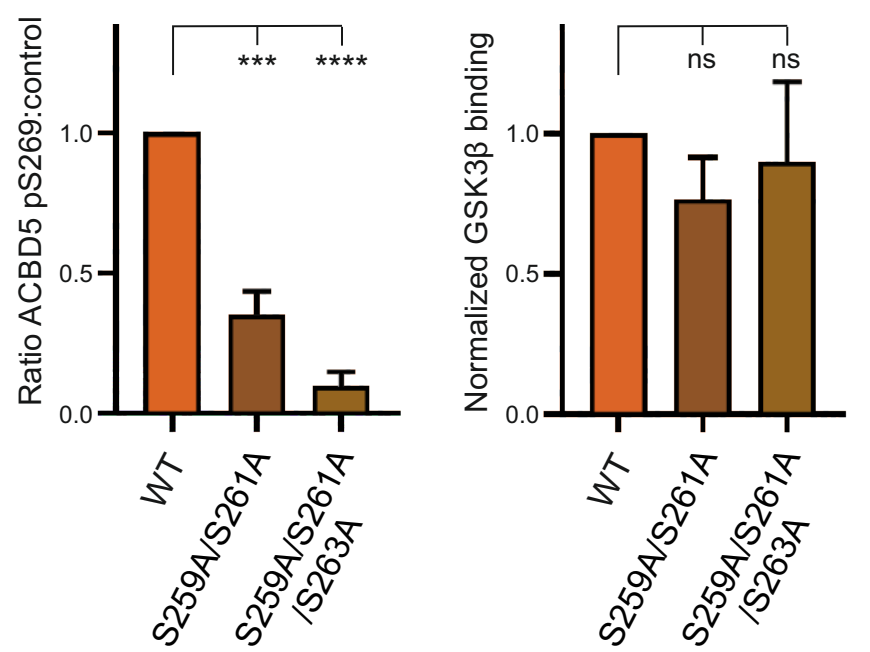
Fiqure 7 bioRxiv preprint doi: https://doi.org/10.1101/2021.11.11.467785; this version posted November 12, 2021. The copyright holder for this preprint (which was not certified by peer review) is the author/funder, who has granted bioRxiv a license to display the preprint in perpetuity. It is made available under aCC-BY-NC-ND 4.0 International license.

A

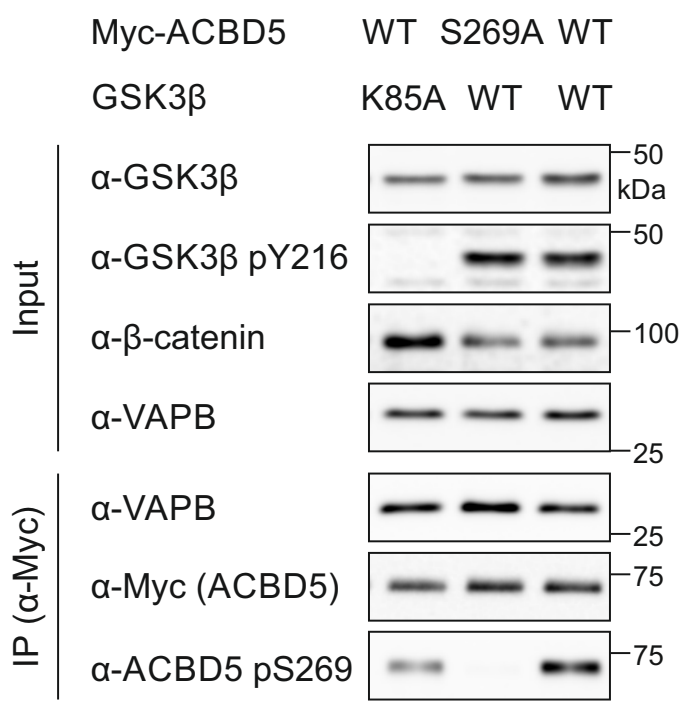

B

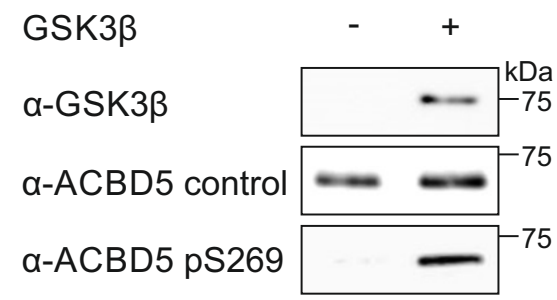

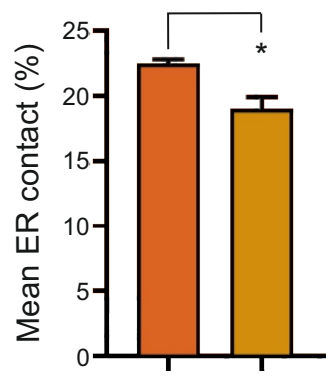

GSK3ß K85A WT

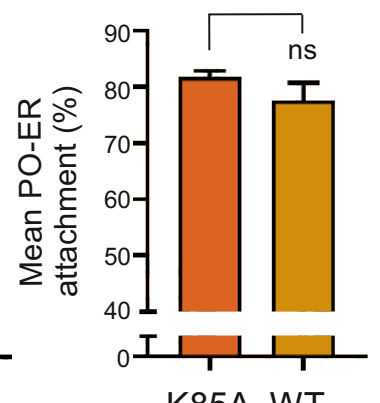

K85A WT

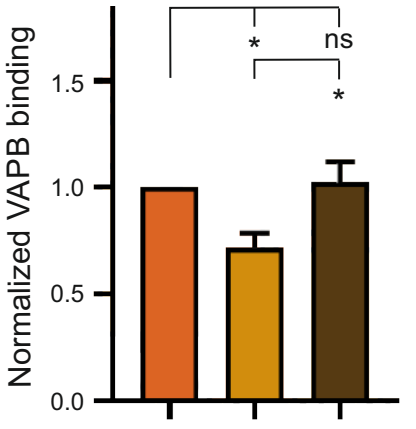

Myc-ACBD5 WT WT S269A GSK3ß K85A WT WT
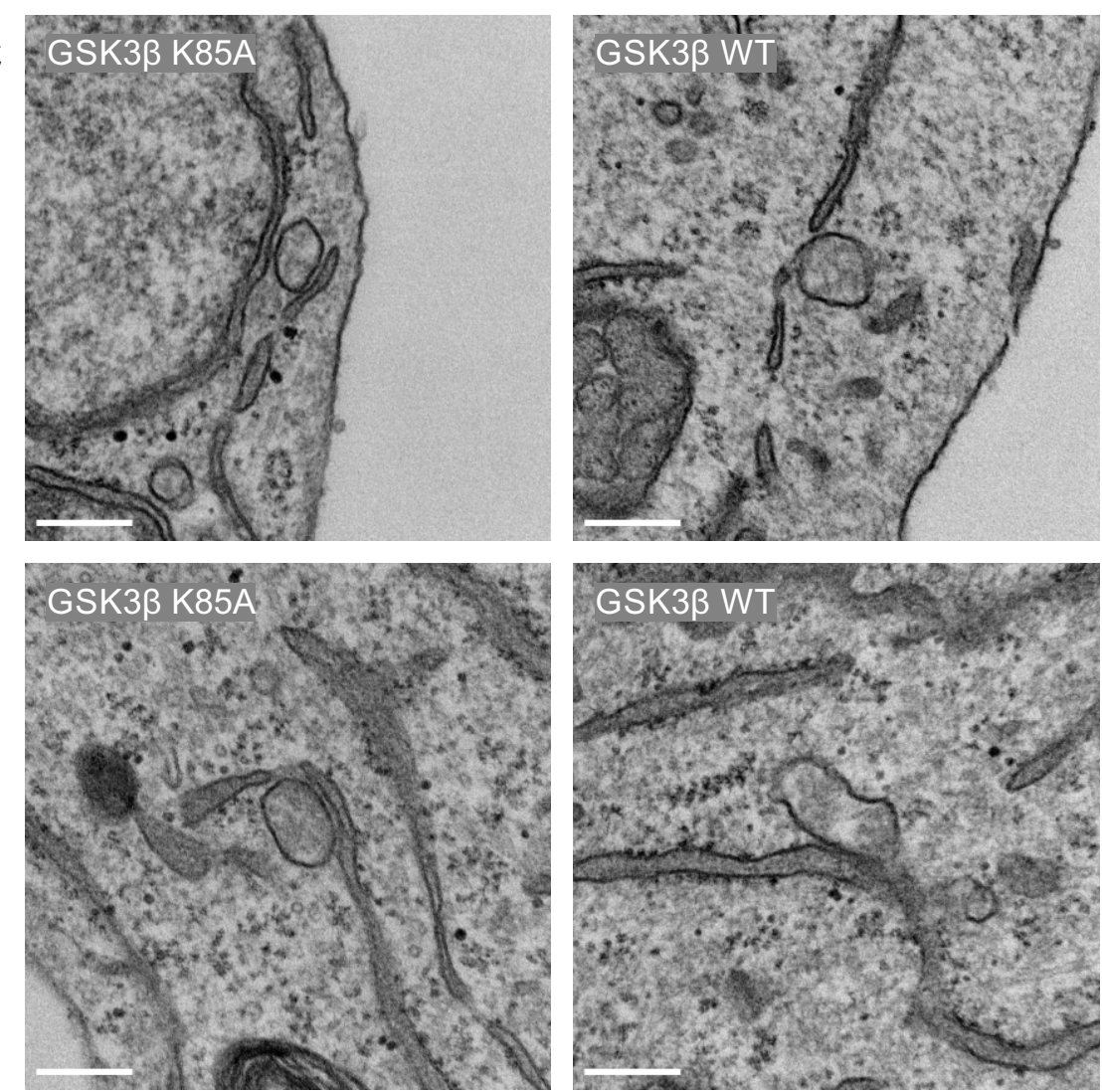
Fiqure 8 bioRxiv preprint doi: https://doi.org/10.1101/2021.11.11.467785; this version posted November 12, 2021. The copyright holder for this preprint (which was not certified by peer review) is the author/funder, who has granted bioRxiv a license to display the preprint in perpetuity. It is made available under aCC-BY-NC-ND 4.0 International license.

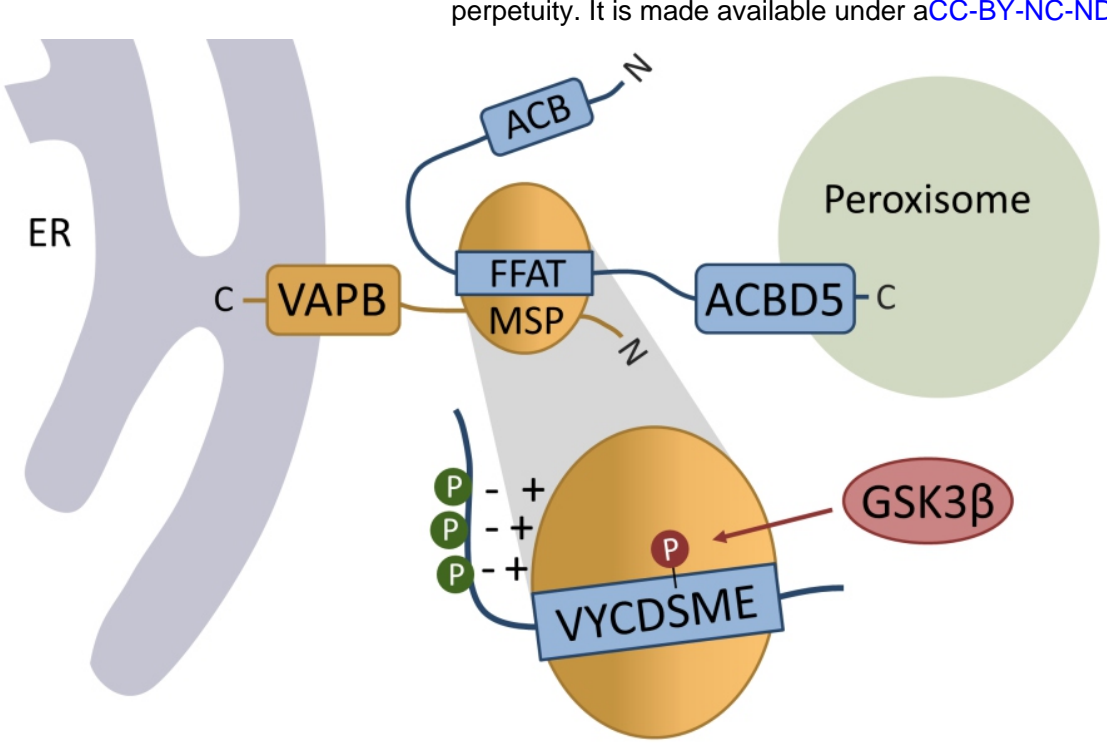

